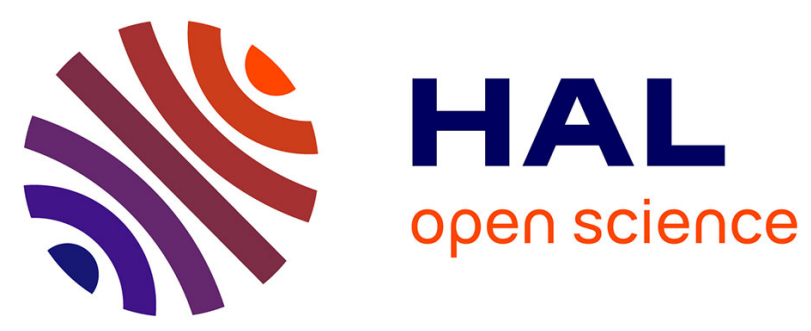

\title{
Monte-Carlo simulation of colliding particles or coalescing droplets transported by a turbulent flow in the framework of a joint fluid-particle pdf approach
} Pascal Fede, Olivier Simonin, Philippe Villedieu

\section{- To cite this version:}

Pascal Fede, Olivier Simonin, Philippe Villedieu. Monte-Carlo simulation of colliding particles or coalescing droplets transported by a turbulent flow in the framework of a joint fluidparticle pdf approach. International Journal of Multiphase Flow, 2015, vol. 74, pp. 165-183. 10.1016/j.ijmultiphaseflow.2015.04.006 . hal-01362106

\section{HAL Id: hal-01362106 https://hal.science/hal-01362106}

Submitted on 8 Sep 2016

HAL is a multi-disciplinary open access archive for the deposit and dissemination of scientific research documents, whether they are published or not. The documents may come from teaching and research institutions in France or abroad, or from public or private research centers.
L'archive ouverte pluridisciplinaire HAL, est destinée au dépôt et à la diffusion de documents scientifiques de niveau recherche, publiés ou non, émanant des établissements d'enseignement et de recherche français ou étrangers, des laboratoires publics ou privés. 


\section{Open Archive TOULOUSE Archive Ouverte (OATAO)}

OATAO is an open access repository that collects the work of Toulouse researchers and makes it freely available over the web where possible.

This is an author-deposited version published in : http://oatao.univ-toulouse.fr/ Eprints ID : 15729

To link to this article : DOI:10.1016/j.ijmultiphaseflow.2015.04.006 URL : $\underline{\text { http://dx.doi.org/10.1016/j.ijmultiphaseflow.2015.04.006 }}$

To cite this version : Fede, Pascal and Simonin, Olivier and Villedieu, Philippe Monte-Carlo simulation of colliding particles or coalescing droplets transported by a turbulent flow in the framework of a joint fluid-particle pdf approach. (2015) International Journal of Multiphase Flow, vol. 74. pp. 165-183. ISSN 0301-9322

Any correspondence concerning this service should be sent to the repository administrator: staff-oatao@,listes-diff.inp-toulouse.fr 


\title{
Monte-Carlo simulation of colliding particles or coalescing droplets transported by a turbulent flow in the framework of a joint fluid-particle pdf approach
}

\author{
Pascal Fede $^{\mathrm{a}, \mathrm{b}, *}$, Olivier Simonin ${ }^{\mathrm{a}, \mathrm{b}}$, Philippe Villedieu $^{\mathrm{c}}$ \\ a Université de Toulouse, INPT, UPS, Institut de Mécanique des Fluides de Toulouse, Allée Camille Soula, FR-31400 Toulouse, France \\ ${ }^{\mathrm{b}} \mathrm{CNRS}$, IMFT, FR-31400 Toulouse, France \\ ${ }^{\mathrm{C}}$ ONERA, DMAE, 2 avenue Edouard Belin, FR-31055 Toulouse, France
}

Keywords:

Statistical description

Monte-Carlo

Collision

Coalescence

Solid particles

Droplets

Turbulence

\begin{abstract}
A B S T R A C T
The aim of the paper is to introduce and validate a Monte-Carlo algorithm for the prediction of an ensemble of colliding solid particles, or coalescing liquid droplets, suspended in a turbulent gas flow predicted by Reynolds Averaged Navier Stokes approach (RANS). The new algorithm is based on the direct discretization of the collision/coalescence kernel derived in the framework of a joint fluid-particle pdf approach proposed by Simonin et al. (2002). This approach allows to take into account correlations between colliding inertial particle velocities induced by their interaction with the fluid turbulence. Validation is performed by comparing the Monte-Carlo predictions with deterministic simulations of discrete solid particles coupled with Direct Numerical Simulation (DPS/DNS), or Large Eddy Simulation (DPS/LES), where the collision/coalescence effects are treated in a deterministic way. Five cases are investigated: elastic monodisperse particles, non-elastic monodisperse particles, binary mixture of elastic particles and binary mixture of elastic settling particles in turbulent flow and finally coalescing droplets. The predictions using the new Monte-Carlo algorithm are in much better agreement with DPS/DNS results than the ones using the standard algorithm.
\end{abstract}

\section{Introduction}

Turbulent gas flows carrying dispersed solid or liquid phases are extensively found in industrial and environmental processes. Some typical examples are fuel spray injection in combustion chamber, solid rocket boosters with alumina droplets, pulverized coal combustion chamber, sediments transport, or rain droplet growth. In such particle- or droplet-laden flows many complex physical phenomena take place such as turbulent dispersion, particle-particle collisions, particle-wall rebounds/impingement, or turbulence modulation by the particles. For example, in a combustion chamber the droplet and gas phase mixing governs the quality and efficiency of the combustion and, consequently, the pollutant emissions. In the near liquid injection zone, droplet coalescence may influence the droplet size distribution and must be accounted for in mathematical models and numerical simulation tools.

The Lagrangian tracking of particles, or droplets, is widely used for the numerical simulation of particle-laden turbulent flows.

* Corresponding author at: Université de Toulouse, INPT, UPS, Institut de Mécanique des Fluides de Toulouse, Allée Camille Soula, FR-31400 Toulouse, France. E-mail address: pascal.fede@imft.fr (P. Fede).
Discrete Particle Simulation (DPS) can be either coupled with Direct Numerical Simulation (DPS/DNS), Large Eddy Simulation (DPS/LES) or Reynolds Averaged Navier-Stokes approach (DPS/RANS) (see for example Balachandar and Eaton (2010), Riber et al. (2009), Fox (2012), and Sommerfeld (2001)). When the collisions are handled by a deterministic algorithm (Discrete Element Method) the DPS/DNS can be considered as a deterministic simulation because no stochastic model for both particle turbulent dispersion and particle collision are needed. For the DPS/LES, a dispersion model to reconstruct the subgrid fluid fluctuating velocity along the particle trajectory can be necessary if the particle relaxation time is of the same order, or smaller than, the characteristic time of the subgrid fluid turbulence (Fede and Simonin, 2006). The DPS/RANS can be stated has stochastic because even if the collisions are not taken into account a stochastic model has to be used for the turbulence induced particle dispersion. In practical applications, due to the huge number of real particles involved, the simultaneous computation of all individual particle trajectories is generally not yet possible. To overcome this difficulty, in the framework of a statistical approach, only a restricted number of numerical particles (also called parcels) may be tracked, each parcel representing a given number of real particles. To account 
for the collisions, stochastic algorithms are then used instead of deterministic ones.

Stochastic algorithms were first derived for the collision of molecules in rarefied gases (Bird, 1969). A few decades ago, in the framework of DPS/RANS approach these algorithms were directly used for taking into account the particle-particle collisions in gas-solid turbulent flow (O'Rourke, 1981; Tanaka and Tsuji, 1991). However, Berlemont et al. (1995) proved that when applied to turbulent two-phase flow the standard stochastic collision algorithms lead to a decrease in particle kinetic energy although the particle collisions are elastics. They identified that this spurious phenomenon is induced by the so-called "molecular chaos" assumption that destroys the fluid-particle covariance and therefore decreases the production of particle fluctuating kinetic energy due to the interaction with fluid turbulence. Sommerfeld (2001) and Berlemont et al. (2001) proposed stochastic approaches where one single particle is tracked and successive random processes are applied to generate fictitious partners of collision accounting for the particle-particle velocity correlations induced by their interaction with fluid turbulence. Their two algorithms differ in the way used for the sampling of the fictitious colliding partner velocity. In order to validate both approaches, simulations have been carried out for homogeneous isotropic flow and they have been compared with DPS/LES from Laviéville et al. (1995) and Gourdel et al. (1998). Even if these single-particle algorithms have been successfully used to simulate two-phase flows, they have several limitations. First, both approaches need an a priori model to sample the fictitious partner velocity, given the velocity of the tracked particle. Second, in contrast with theory and DPS/DNS results, these algorithms do not preserve fluid-particle velocity covariance. To overcome this issue Berlemont et al. (2001) proposed a multiple particle collision algorithm based on the simultaneous tracking of several particles combined with an approximate method to enforce the fluid-particle velocity covariance conservation. This ad-hoc method consists in changing the seen fluid velocity of each colliding particle after a collision in order to ensure that the fluid-particle velocity covariance is conserved. But, as pointed out by Berlemont et al. (2001), this method is not satisfactory because the distribution of collision angles remains the same whatever the particle inertia since no specific correlation is accounting for before the collision. Finally for single-particle methods the conservation of momentum and of the particle kinetic energy (for elastic collision) cannot be exactly ensured because the collision partner is fictitious. In fact, such algorithms are statistically conservative only if the number of parcels is very large.

In the present paper, we propose a rigorous approach to derive a Monte-Carlo algorithm which allows to overcome all the previously mentioned limitations in the framework of DPS/RANS approach. This algorithm can be interpreted as a direct discretization of the collision kernel introduced in Laviéville et al. (1995, 1997) and Simonin et al. (2002) for taking into account the velocity correlations induced by the interactions of particles with turbulence. For the sake of completeness the paper first introduces the full derivation of the joint fluid-particle Number Density Function (NDF) kinetic equation. The case of solid particles, and liquid droplets, are both addressed. The standard and the new Monte-Carlo algorithms for solving the kinetic equation are described in the third section. In this section an analysis of the effect of the numerical parameters introduced by the novel Monte-Carlo algorithm is performed. Section 'Monodisperse solid particles' shows the results obtained by considering a monodisperse elastic, and non-elastic, particles suspended in homogeneous isotropic turbulent flow. Section 'Binary mixture of colliding particles' is dedicated to binary mixture of particles suspended, and settling, in homogeneous isotropic turbulence and Section Coalescing droplets shows the application of the modified Monte-Carlo algorithm for coalescing liquid droplets transported in a homogeneous isotropic turbulent flow.

\section{Statistical description of binary collision between particles transported by a turbulent flows}

\section{Statistical description}

The statistical description of a dispersed phase, composed of solid particles or droplets, transported by a turbulent fluid flow relies on the analogy with the thermal motion of molecules as described by the kinetic theory of rarefied gases (Chapman and Cowling, 1970). In this framework, the dispersed phase statistical properties are described by the particle number density function $f_{p}\left(\mathbf{c}_{p}, \mu_{p} ; \mathbf{x}, t\right)$ defined such that $f_{p}\left(\mathbf{c}_{p}, \mu_{p} ; \mathbf{x}, t\right) d \mathbf{c}_{p} d \mu_{p} d \mathbf{x}$ is the mean probable number of particles at time $t$ with a centre of mass located in the volume $[\mathbf{x}, \mathbf{x}+d \mathbf{x}]$, having a mass $m_{p}$ in $\left[\mu_{p}, \mu_{p}+d \mu_{p}\right]$ and a translation velocity $\mathbf{u}_{p}$ in $\left[\mathbf{c}_{p}, \mathbf{c}_{p}+d \mathbf{c}_{p}\right]$. By analogy with statistical approaches for single-phase turbulent flows, the statistical average associated to the definition of $f_{p}$ may be defined as an ensemble average on an infinite number of realizations of a given gas-particle flow (Buyevich, 1971, 1972). The Number Density Function (NDF), also called in the literature the probability density function (PDF), obeys to a Boltzmann-like kinetic equation. However, in contrast to the thermal motion of molecules or to dry granular flows, the particle, or droplet, motion is driven by the fluid turbulence, and the influence of the fluid turbulent flow on the particle dynamics must be taken into account. The closure of the term representing the forces acting on the particles is the topic of many studies for taking into account the turbulent dispersion by the fluid turbulence (Derevich and Zaichik, 1988; Reeks, 1992, 1993). Simonin (1996) proposed an original statistical description taking into account the instantaneous fluid velocity seen by the particles. A joint fluid-particle distribution $f_{f p}\left(\mathbf{c}_{p}, \mathbf{c}_{f}, \mu_{p} ; \mathbf{x}, t\right)$ is then introduced, which is defined such that $f_{f p}\left(\mathbf{c}_{p}, \mathbf{c}_{f}, \mu_{p} ; \mathbf{x}, t\right) d \mathbf{c}_{p} d \mathbf{c}_{f} d \mu_{p} d \mathbf{x}$ is the mean probable number of particles at time $t$ with a centre of mass located in the volume $[\mathbf{x}, \mathbf{x}+d \mathbf{x}]$, and having a mass $m_{p}$ in $\left[\mu_{p}, \mu_{p}+d \mu_{p}\right]$, a translation velocity $\mathbf{u}_{p}$ in $\left[\mathbf{c}_{p}, \mathbf{c}_{p}+d \mathbf{c}_{p}\right]$ and "seeing" a fluid velocity $\mathbf{u}_{f @ p}$ in $\left[\mathbf{c}_{f}, \mathbf{c}_{f}+d \mathbf{c}_{f}\right]$. In the framework of Gatignol (1983) or Maxey and Riley (1983) approach the fluid velocity $\mathbf{u}_{f @ p}$ represents the local undisturbed fluid velocity at the particle position introduced to model the fluid-particle momentum transfer.

In the case without mass transfer meaning that the change of any particle mass is only due to collision, Simonin (1996) proposed the following Boltzmann-like transport equation for the joint fluid-particle distribution

$$
\begin{gathered}
\frac{\partial f_{f p}}{\partial t}+\frac{\partial}{\partial x_{i}}\left[c_{p, i} f_{f p}\right]+\frac{\partial}{\partial c_{p, i}}\left[\left\langle\frac{d u_{p, i}}{d t} \mid \mathbf{c}_{p}, \mathbf{c}_{f}\right\rangle f_{f p}\right] \\
+\frac{\partial}{\partial c_{f, i}}\left[\left\langle\frac{d u_{f, i}}{d t} \mid \mathbf{c}_{p}, \mathbf{c}_{f}\right\rangle f_{f p}\right]=\left(\frac{\partial f_{f p}}{\partial t}\right)_{\text {coll }}
\end{gathered}
$$

where $\left\langle\cdot \mid \mathbf{c}_{p}, \mathbf{c}_{f}\right\rangle$ is the ensemble average conditioned by the particle and fluid velocity seen by any particle with a centre of mass at position $\mathbf{x}_{p}=\mathbf{x}: \mathbf{u}_{p}=\mathbf{c}_{p}$ and $\mathbf{u}_{f @ p}=\mathbf{c}_{f}$ (with $\mathbf{u}_{p}$ and $\mathbf{u}_{f @ p}$ being the particle and the fluid velocity seen by the particle in physical space). The term on the right-hand side of Eq. (1) represents the change of $f_{f p}$ due to collision, coalescence or break-up. The third term on the left-hand side of Eq. (1) accounts for the effects of the particle acceleration on the NDF. Considering that the forces acting on the particles are only the gravity and the drag force, the particle acceleration writes 
$\frac{d \mathbf{u}_{p}}{d t}=\frac{\mathbf{F}_{p}}{m_{p}}=-\frac{\mathbf{u}_{p}-\mathbf{u}_{f @ p}}{\tau_{p}}+\mathbf{g}$

where $\mathbf{u}_{f @ p}$ is the locally undisturbed fluid velocity at the particle position, or the so-called fluid velocity "seen" by the particle, $\mathbf{g}$ the gravity and $\tau_{p}$ the local instantaneous particle response time. Compared to the standard approach based on $f_{p}\left(\mathbf{c}_{p} ; \mathbf{x}, t\right)$, the particle acceleration term (the third term of Eq. (1)) is directly closed because the instantaneous fluid velocity is known through the joint fluid-particle NDF. However, the price-to-pay is the presence of the fourth term representing the acceleration of the fluid velocity along the solid particle trajectory. Several models can be found in the literature dedicated to the Lagrangian modelling of the fluid turbulence along fluid elements (Haworth and Pope, 1986; Pope, 1994, 2002) or along inertial particle trajectories (Simonin et al., 1993; Pascal and Oesterlé, 2000; Minier and Peirano, 2001; Minier et al., 2004; Pialat et al., 2007; Tanière et al., 2010). As the purpose of the present paper is the numerical treatment of collision, the Lagrangian prediction of the fluid velocity along a particle trajectory will not be longer detailed. In the present paper, the fluid flow, for stochastic simulations, is predicted by a Langevin equation proposed by Simonin et al. (1993) that is an extension of the model proposed by Pope (1994) (see Appendix B).

\section{Collision/coalescence kernel}

The collision/coalescence operator may be split in two contributions

$$
\left(\frac{\partial f_{f p}}{\partial t}\right)_{\text {coll }}=K^{+}\left(\mathbf{c}_{p}, \mathbf{c}_{f @ p}, \mu_{p} ; \mathbf{x}, t\right)-K^{-}\left(\mathbf{c}_{p}, \mathbf{c}_{f @ p}, \mu_{p} ; \mathbf{x}, t\right)
$$

where $K^{+}\left(\mathbf{c}_{p}, \mathbf{c}_{f @ p}, \mu_{p} ; \mathbf{x}, t\right)$ represents the apparition rate and $K^{-}\left(\mathbf{c}_{p}, \mathbf{c}_{f @ p}, \mu_{p} ; \mathbf{x}, t\right)$ the vanishing rate. Both terms require the knowledge of the velocity and mass for the two colliding particles. These information are given by the pair NDF $f_{f p}^{(2)}$ defined such that

$f_{f p}^{(2)}\left(\mathbf{c}_{p}, \mathbf{c}_{f @ p}, \mu_{p}, \mathbf{c}_{q}, \mathbf{c}_{f @ q}, \mu_{q} ; \mathbf{x}_{p}, \mathbf{x}_{q}, t\right) d \mathbf{x}_{p} d \mathbf{x}_{q} d \mathbf{c}_{p} d \mathbf{c}_{q} d \mathbf{c}_{f @ p} d \mathbf{c}_{f @ q} d \mu_{p} d \mu_{q}$

is the mean probable number of particle pairs with centres of mass located in the volume $\left[\mathbf{x}_{p}, \mathbf{x}_{p}+d \mathbf{x}_{p}\right]$ and $\left[\mathbf{x}_{q}, \mathbf{x}_{q}+d \mathbf{x}_{q}\right]$ and having masses $m_{p}$ in $\left[\mu_{p}, \mu_{p}+d \mu_{p}\right]$ and $m_{q}$ in $\left[\mu_{q}, \mu_{q}+d \mu_{q}\right]$, and translation velocities $\mathbf{u}_{p}$ in $\left[\mathbf{c}_{p}, \mathbf{c}_{p}+d \mathbf{c}_{p}\right]$ and $\mathbf{u}_{q}$ in $\left[\mathbf{c}_{q}, \mathbf{c}_{q}+d \mathbf{c}_{q}\right]$, and viewing fluid velocities $\mathbf{u}_{f @ p}$ in $\left[\mathbf{c}_{f @ p}, \mathbf{c}_{f @ p}+d \mathbf{c}_{f @ p}\right]$ and $\mathbf{u}_{f @ q}$ in $\left[\mathbf{c}_{f @ q}, \mathbf{c}_{f @ q}+d \mathbf{c}_{f @ q}\right]$, respectively. From this definition the vanishing rate of droplets writes

$$
\begin{aligned}
K^{-}\left(\mathbf{c}_{p}, \mathbf{c}_{f @ p}, \mu_{p} ; \mathbf{X}, t\right)= & \int_{\mathbf{w}_{p q} \cdot \mathbf{k}_{p q}<0} f_{f p}^{(2)}\left(\mathbf{c}_{p}, \mathbf{c}_{f @ p}, \mu_{p}, \mathbf{c}_{q}, \mathbf{c}_{f @ q}, \mu_{q} ; \mathbf{X}, \mathbf{X}+d_{p q} \mathbf{k}_{p q}, t\right) \\
& \times\left|\mathbf{w}_{p q} \cdot \mathbf{k}_{p q}\right| d_{p q}^{2} d \mathbf{c}_{q} d \mathbf{c}_{f @ q} d \mu_{q} d \mathbf{k}_{p q}
\end{aligned}
$$

where the particle-particle relative velocity is defined as $\mathbf{w}_{p q}=\mathbf{c}_{q}-\mathbf{c}_{p}$. The impact vector is defined by the particle position $\mathbf{k}_{p q}=\left(\mathbf{x}_{q}-\mathbf{x}_{p}\right) / d_{p q}$ and the collision diameter $d_{p q}=\left(d_{p}+d_{q}\right) / 2$. The rate of particle apparition is then given by

$$
\begin{aligned}
K^{+}\left(\mathbf{c}_{p}, \mathbf{c}_{f @ p}, \mu_{p} ; \mathbf{x}, t\right)= & \int_{\mathbf{w}_{p q}, \mathbf{k}_{p q}<0} R\left(\mathbf{c}_{m}, \mathbf{c}_{f @ m}, \mu_{m}, \mathbf{c}_{n}, \mathbf{c}_{f @ n}, \mu_{n}, \mathbf{k}_{m n} \rightarrow \mathbf{c}_{p}, \mathbf{c}_{f @ p}, \mu_{p} ; \mathbf{x}, t\right) \\
& \times f_{f p}^{(2)}\left(\mathbf{c}_{m}, \mathbf{c}_{f @ m} \mu_{m}, \mathbf{c}_{n}, \mathbf{c}_{f @ n}, \mu_{n} ; \mathbf{x}, \mathbf{x}+d_{m n} \mathbf{k}_{m n}, t\right) \\
& \times\left|\mathbf{w}_{m n} \cdot \mathbf{k}_{m n}\right| d_{m n}^{2} d \mathbf{c}_{n} d \mathbf{c}_{f @ n} d \mu_{n} d \mathbf{k}_{m n} d \mathbf{c}_{m} d \mathbf{c}_{f @ m} d \mu_{m}
\end{aligned}
$$

where the transition probability by particle-particle collision $R\left(\mathbf{c}_{m}, \mathbf{c}_{f @ m}, \mu_{m}, \mathbf{c}_{n}, \mathbf{c}_{f @ n}, \mu_{n}, \mathbf{k}_{m n} \rightarrow \mathbf{c}_{p}, \mathbf{c}_{f @ p}, \mu_{p} ; \mathbf{x}, t\right)=P\left(\mathbf{c}_{p}, \mathbf{c}_{f @ p}, \mu_{p} \mid \mathbf{c}_{m}\right.$, $\left.\mathbf{c}_{f @ m}, \mu_{m}, \mathbf{c}_{n}, \mathbf{c}_{f @ n}, \mu_{n}, \mathbf{k}_{m n} ; \mathbf{x}, t\right)$ is introduced. This function is the probability density of having one particle located at $\mathbf{x}$ with a velocity $\mathbf{c}_{p}$ and mass $\mu_{p}$ resulting from the collision of a particle $m$ located in $\mathbf{x}_{m}=\mathbf{x}$ with a velocity $\mathbf{c}_{m}$, and a mass $\mu_{m}$, with a particle $n$ located in $\mathbf{x}_{n}=\mathbf{x}+d_{m n} \mathbf{k}_{m n}$ with a velocity $\mathbf{c}_{n}$ and a mass $\mu_{n}$. Considering the hard-sphere collision model, the transition probability density reads:

$$
\begin{aligned}
& P\left(\mathbf{c}_{p}, \mathbf{c}_{f @ p}, \mu_{p} \mid \mathbf{c}_{m}, \mathbf{c}_{f @ m}, \mu_{m}, \mathbf{c}_{n}, \mathbf{c}_{f @ n}, \mu_{n}, \mathbf{k}_{m n} ; \mathbf{x}, t\right) \\
& \quad=\delta\left[\mu_{p}-\mu_{m}\right] \times \delta\left[\mathbf{c}_{p}-\mathbf{c}_{m}^{+}\right] \times P\left(\mathbf{c}_{f @ p} \mid \mathbf{c}_{f @ m}, \mathbf{c}_{f @ n}\right)
\end{aligned}
$$

where $\mathbf{c}_{m}^{+}$is the $m$-particle velocity after an inelastic collision with any $n$-particle. Assuming, frictionless hard-sphere collision, these velocities are given by:

$$
\begin{aligned}
& \mathbf{c}_{m}^{+}=\mathbf{c}_{m}+\frac{m_{n}}{m_{m}+m_{n}}\left(1+e_{c}\right)\left(\mathbf{w}_{m n} \cdot \mathbf{k}_{m n}\right) \mathbf{k}_{m n}, \\
& \mathbf{c}_{n}^{+}=\mathbf{c}_{n}-\frac{m_{m}}{m_{m}+m_{n}}\left(1+e_{c}\right)\left(\mathbf{w}_{m n} \cdot \mathbf{k}_{m n}\right) \mathbf{k}_{m n}
\end{aligned}
$$

where $e_{c}$ is the particle-particle restitution coefficient (Walton, 1993).

When considering the collision of two droplets several phenomena may occur as pure coalescence, elastic bouncing, or production of satellite droplets (Ashgriz and Poo, 1990; Crowe et al., 1998). In the present study, only the permanent coalescence regime where a droplet-droplet collision leads to a new droplet is considered (Villedieu and Simonin, 2004). The mass and momentum conservation leads to the two following relations

$\mu_{p}=\mu_{m}+\mu_{n}$

$\mu_{p} \mathbf{c}_{p}=\mu_{m} \mathbf{c}_{m}+\mu_{n} \mathbf{c}_{n}$.

Then the transition probability density can be written as

$$
\begin{aligned}
& P\left(\mathbf{c}_{p}, \mathbf{c}_{f @ p}, \mu_{p} \mid \mathbf{c}_{m}, \mathbf{c}_{f @ m}, \mu_{m}, \mathbf{c}_{n}, \mathbf{c}_{f @ n}, \mu_{n}, \mathbf{k}_{m n} ; \mathbf{x}, t\right) \\
& =\frac{1}{2} \delta\left[\mu_{p}-\left(\mu_{m}+\mu_{n}\right)\right] \times \delta\left[\mathbf{c}_{p}-\frac{\mu_{m} \mathbf{c}_{m}+\mu_{n} \mathbf{c}_{n}}{\mu_{m}+\mu_{n}}\right] \\
& \quad \times P\left(\mathbf{c}_{f @ p} \mid \mathbf{c}_{f @ m}, \mathbf{c}_{f @ n}\right)
\end{aligned}
$$

the coefficient $1 / 2$ is chosen to represent that the resulting droplet is either located in $\mathbf{x}$ or $\mathbf{x}+d_{m n} \mathbf{k}_{m n}$ with the same probability density. In Eq. (11), $P\left(\mathbf{c}_{f @ p} \mid \mathbf{c}_{f @ m}, \mathbf{c}_{f @ n}\right)$ is the probability that the fluid velocity seen by the children droplet is $\mathbf{c}_{f @ p}$ knowing that the two fluid velocities seen by the coalescing droplets are $\mathbf{c}_{f @ m}$ and $\mathbf{c}_{f @ n}$. Assuming that the two colliding droplets have a diameter smaller than the integral length scale of the turbulence $\left(d_{p} \ll L_{f}\right)$ then the coalescing droplets should "see" nearly the same fluid velocity and consequently

$P\left(\mathbf{c}_{f @ p} \mid \mathbf{c}_{f @ m}, \mathbf{c}_{f @ n}\right)=\delta\left[\mathbf{c}_{f @ p}-\mathbf{c}_{f @ m}\right]=\delta\left[\mathbf{c}_{f @ p}-\mathbf{c}_{f @ n}\right]$.

In case of solid particles, by definition the fluid velocity seen by the particles is unchanged by the collision, consequently $\mathbf{c}_{f @ m}=\mathbf{c}_{f @ p}$ (Laviéville et al., 1997; Singh et al., 2004).

\section{Pair number distribution function closure}

In the framework of the standard kinetic theory of dense gases (Chapman and Cowling, 1970), the pair NDF is written as the product of single NDF,

$f_{p}^{(2)}\left(\mathbf{c}_{p}, \mathbf{c}_{q} ; \mathbf{x}, \mathbf{x}+d_{p q} \mathbf{k}_{p q}, t\right)=g_{0} f_{p}\left(\mathbf{c}_{p} ; \mathbf{x}, t\right) f_{p}\left(\mathbf{c}_{q} ; \mathbf{x}+d_{p q} \mathbf{k}_{p q}, t\right)$

The radial distribution function, $g_{0}$, takes into account the particle spatial correlation effects. In the framework of the kinetic theory of granular media, $g_{0}$, has been introduced to take into account the increase of the collision frequency when the solid volume fraction is approaching the maximum packing (Jenkins and Richman, 1985; Lun and Savage, 1986). At the opposite, for dilute flows, as in the present paper, this effect is negligible and the radial distribution function should be approximated by 1 . However, in turbulent 
two-phase flows, the interaction of inertial particles with turbulence may induce accumulation of particles in low-vorticity regions. This phenomenon, called preferential concentration, leads to values of $g_{0}$ larger than unity (Sundaram and Collins, 1997; Shaw et al., 1998; Reade and Collins, 2000; Salazar et al., 2008). Due to the complexity of the physical mechanisms taking place in preferential concentration, the modelling of the radial distribution function with respect to the particle inertia is still an open issue. Restricting our attention to inertial particles with relaxation time larger than the Kolmogorov time scale the radial distribution function is approximated by 1 leading to an underestimation of the collision frequency. In addition, in dilute flow the spatial variations of the NDF are assumed to be negligible on a length scale of the order of the particle diameter. Therefore the position of the particles in right-hand side of Eq. (13) can be assumed identical. In the frame of the above assumptions for dilute flows the pair NDF may be written as

$f_{p}^{(2)}\left(\mathbf{c}_{p}, \mathbf{c}_{q} ; \mathbf{x}, \mathbf{x}+d_{p q} \mathbf{k}_{p q}, t\right)=f_{p}\left(\mathbf{c}_{p} ; \mathbf{x}, t\right) f_{p}\left(\mathbf{c}_{q} ; \mathbf{x}, t\right)$

Eq. (14), often called molecular chaos assumption, means that the colliding particles have independent velocities. Indeed, even for separation smaller than the Kolmogorov length scale, particle inertia should maintain the influence of the interactions with fluid elements at larger separation which are only partially correlated. According to Abrahamson (1975), when the particle relaxation time is much larger than the turbulent characteristic macro time scale, the velocities of colliding particles should be almost uncorrelated and the molecular assumption is truly relevant. In contrast, when the particle response time is of the same order, or smaller, than the turbulent characteristic macro time scale of the turbulence (such as the Eulerian or Lagrangian integral time scales), the approaching particle velocities are influenced by the same local gas turbulent flow. Hence colliding particle velocities are correlated, via the gas-particle interaction, and particle-particle relative velocity decreases (Williams and Crane, 1983; Kruis and Kuster, 1997). This effect has been already scrutinized by DPS/DNS and DPS/LES in case homogeneous isotropic turbulent flow carrying a monodisperse (Laviéville et al., 1995) and a binary mixture Fede and Simonin, 2003 of inertial particles; but also in case of simple shear flow (Laviéville et al., 1997). To overcome this limitation, Laviéville et al. (1995) proposed an original decomposition of the pair NDF. Hereafter, for the sake of simplicity, time and position variables are both skipped. Using conditional expectation, the authors decomposed the pair joint NDF as $f_{f p}^{(2)}\left(\mathbf{c}_{p}, \mathbf{c}_{f @ p}, \mathbf{c}_{q}, \mathbf{c}_{f @ q}\right)=$ $P_{f}^{(2)}\left(\mathbf{c}_{f @ p}, \mathbf{c}_{f @ q}\right) f_{p}^{(2)}\left(\mathbf{c}_{p}, \mathbf{c}_{q} \mid \mathbf{c}_{f @ p}, \mathbf{c}_{f @ q}\right)$ and they obtained

$f_{f p}^{(2)}\left(\mathbf{c}_{p}, \mathbf{c}_{f @ p}, \mathbf{c}_{q}, \mathbf{c}_{f @ q}\right)=P_{f}^{(2)}\left(\mathbf{c}_{f @ p}, \mathbf{c}_{f @ q}\right) \times f_{p}\left(\mathbf{c}_{q} \mid \mathbf{c}_{f @ p}, \mathbf{c}_{f @ q}\right) f_{p}\left(\mathbf{c}_{p} \mid \mathbf{c}_{q}, \mathbf{c}_{f @ p}, \mathbf{c}_{f @ q}\right)$

For closing this relationship, according to the discussion above on the physical mechanism controlling the correlation effect between neighbouring particles, Laviéville et al. (1995) assume that the dependency of a particle velocity $\mathbf{c}_{p}$ probability on the $q$-particle and gas velocity realizations is mainly accounted for via the conditioning by the fluid velocities "seen", leading to $f_{p}\left(\mathbf{c}_{p} \mid \mathbf{c}_{q}, \mathbf{c}_{f @ p}, \mathbf{c}_{f @ q}\right) \approx f_{p}\left(\mathbf{c}_{p} \mid \mathbf{c}_{f @ p}, \mathbf{c}_{f @ q}\right)$. Finally, if the particle diameter is much smaller than the turbulent integral correlation length scale, the two particles nearly "see" the same fluid velocity. Then Laviéville et al. (1995) write the pair NDF as

$f_{f p}^{(2)}\left(\mathbf{c}_{p}, \mathbf{c}_{f @ p}, \mathbf{c}_{q}, \mathbf{c}_{f @ q}\right) \approx \frac{\delta\left[\mathbf{c}_{f @ p}-\mathbf{c}_{f @ q}\right]}{P_{f}\left(\mathbf{c}_{f @ p}\right)} f_{f p}\left(\mathbf{c}_{p}, \mathbf{c}_{f @ p}\right) f_{f p}\left(\mathbf{c}_{q}, \mathbf{c}_{f @ q}\right)$

This equation is obtained by using $f_{f p}\left(\mathbf{c}_{p}, \mathbf{c}_{f @ p}\right)=f_{p}\left(\mathbf{c}_{p} \mid \mathbf{c}_{f @ p}\right) P_{f}\left(\mathbf{c}_{f @ p}\right)$.

In case of homogeneous isotropic turbulent flows the fluid velocity distribution function, $P_{f}\left(\mathbf{c}_{f} @ p\right)$, can be approximated by a Gaussian distribution
$P_{f}\left(\mathbf{c}_{f @ p}\right)=\frac{1}{\left(4 / 3 \pi q_{f @ p}^{2}\right)^{3 / 2}} \exp \left[-\frac{\mathbf{c}_{f @ p}^{2}}{4 / 3 q_{f @ p}^{2}}\right]$

where $q_{f @ p}^{2}$ is the fluid turbulent kinetic energy "seen" by the p-particles which is assumed to be equal to the "true" fluid turbulent kinetic energy $q_{f}^{2}$. It can be noticed that the closure represented by Eq. (16) has been employed to represent the collision effect in Eulerian particle moment equations derived in the framework of the joint-fluid particle NDF approach (Laviéville et al., 1997; Simonin et al., 2002; Fede and Simonin, 2003; Zaichik et al., 2003, 2006).

The assumptions made to get Eq. (16) could lead to an underestimation of the correlation between the two colliding particle velocities. Then the particle-particle relative velocity could be overestimated and consequently the collision frequency is expected to be overestimated Simonin et al. (2002). However, two opposite effects are taking place which influence the model prediction accuracy. On the one hand, the collision frequency is underestimated because the spatial distribution effect is underestimated by taking the radial distribution function, $g_{0}$, equal to 1 . On the another hand, the collision frequency is overestimated because the velocity correlation effect induced by the interaction with the turbulent flow is underestimated. This balancing effect has been pointed out by Zaichik et al. $(2003,2006)$ showing that the model underestimation of the particle collision frequency may be important at small particle inertia, for particle relaxation time of the order of the Kolmogorov time scale, when the segregation mechanism is maximum. In contrast, the model overestimation becomes effective when the particle relaxation time is comparable with the gas turbulent integral time scale.

With Eq. (16) the Boltzmann-like transport equation is closed because the collision kernel writes

$$
\begin{aligned}
K^{-}\left(\mathbf{c}_{p}, \mathbf{c}_{f @ p}, \mu_{p} ; \mathbf{x}, t\right)=\int_{\mathbf{w}_{p q} \cdot \mathbf{k}_{p q}<0} \frac{\delta\left[\mathbf{c}_{f @ p}-\mathbf{c}_{f @ q}\right]}{P_{f}\left(\mathbf{c}_{f @ p}\right)} f_{f p}\left(\mathbf{c}_{p}, \mathbf{c}_{f @ p}\right) f_{f p}\left(\mathbf{c}_{q}, \mathbf{c}_{f @ q}\right) \\
\times\left|\mathbf{w}_{p q} \cdot \mathbf{k}_{p q}\right| d_{p q}^{2} d \mathbf{c}_{q} d \mathbf{c}_{f @ q} d \mu_{q} d \mathbf{k}_{p q} \\
K^{+}\left(\mathbf{c}_{p}, \mathbf{c}_{f @ p}, \mu_{p} ; \mathbf{X}, t\right)=\int_{\mathbf{w}_{p q} \cdot \mathbf{k}_{p q}<0} R \times \frac{\delta\left[\mathbf{c}_{f @ m}-\mathbf{c}_{f @ n}\right]}{P_{f}\left(\mathbf{c}_{f @ m}\right)} f_{f p}\left(\mathbf{c}_{m}, \mathbf{c}_{f @ m}\right) f_{f p}\left(\mathbf{c}_{n}, \mathbf{c}_{f @ n}\right) \\
\times\left|\mathbf{w}_{m n} \cdot \mathbf{k}_{m n}\right| d_{m n}^{2} d \mathbf{c}_{n} d \mathbf{c}_{f @ n} d \mu_{n} d \mathbf{k}_{m n} d \mathbf{c}_{m} d \mathbf{c}_{f @ m} d \mu_{m}
\end{aligned}
$$

where $R \equiv R\left(\mathbf{c}_{m}, \mathbf{c}_{f @ m}, \mu_{m}, \mathbf{c}_{n}, \mathbf{c}_{f @ n}, \mu_{n}, \mathbf{k}_{m n} \rightarrow \mathbf{c}_{p}, \mathbf{c}_{f @ p}, \mu_{p} ; \mathbf{x}, t\right)$ is the probability of transition written without the variables for the sake of simplicity. The following section is dedicated to Monte-Carlo algorithms for solving the kinetic equation. The following section is dedicated to Monte-Carlo algorithms for solving the kinetic equation with the molecular chaos assumption (13) or with the correlated colliding velocity closure (16).

\section{Monte-Carlo algorithm for solving the joint fluid-particle kinetic equation}

\section{Principle of standard Monte-Carlo algorithm}

Stochastic particle methods, also called Monte-Carlo methods, were initially developed for solving the Boltzmann equation for rarefied gas dynamics (Bird, 1969; Nanbu, 1983; Babovsky, 1986; Ivanov and Rogasinsky, 1988). The basic principle of such methods is to approximate the NDF by a linear combination of Dirac masses:

$f_{p}\left(\mathbf{c}_{p} ; \mathbf{x}, t\right)=\sum_{i=1}^{N_{p a r}(t)} w_{p}^{i} \delta\left[\mathbf{x}-\mathbf{x}_{p}^{i}\right] \times \delta\left[\mathbf{c}_{p}-\mathbf{u}_{p}^{i}\right]$

In this sum, each term is generally called "numerical particle" or "parcel" and is interpreted as representing a group of $w_{p}^{i}$ real 
particles located around the same space position, and having the same velocity. The second key feature of stochastic particle methods is the use of a fractional time step algorithm, which consists in splitting each time step in the two following substeps:

- a transport substep which corresponds to the discretization of the non-collisional kinetic equation. During this substep the position, velocity of each numerical particle are updated by solving the particle motion equations.

- a collision substep which corresponds to the discretization of the collision term. Since the calculation of collision probabilities involves the mean number of particle pairs located in an infinitesimal volume centred around any given point, the approximation of the NDF given by Eq. (19) is not compatible with the closure model Eq. (14) for the pair NDF. Indeed, according to Eq. (19) two given particles are either located at the same point with the same velocity (when they belong to the same parcel) or are located at two separated points (when they belong to two different parcels). To overcome this issue, it is assumed that mesh cells are small enough for the exact NDF to be almost uniform over them so that Eq. (19) could be replaced by:

$$
f_{p}\left(\mathbf{c}_{p} ; \mathbf{x}, t\right)=\sum_{C_{i}} \sum_{k \in \operatorname{Ind}\left(C_{i}\right)} w_{p}^{k} \frac{\mathbf{1}_{C_{i}}(\mathbf{x})}{\operatorname{vol}\left(C_{i}\right)} \delta\left[\mathbf{c}_{p}-\mathbf{u}_{p}^{k}\right]
$$

where $C_{i}$ denotes a given cell of the mesh $\mathcal{M}_{x}, \mathbf{1}_{C_{i}}$ is the indicator function of $C_{i}$ (top-hat distribution function), $\operatorname{Ind}\left(C_{i}\right)$ is the list of parcels located in cell $C_{i}$ at the end of the transport step and $\operatorname{vol}\left(C_{i}\right)$ is the volume of $C_{i}$. From a physical point of view, this new approximation of the NDF means that during the collision substep, all real particles represented by a given parcel are supposed to be randomly distributed in the cell containing the parcel instead of being all located at the same point as during the transport step. Of course, this is not mandatory and an independent (moving) mesh could be used as well for the regularization of the NDF. But, what is important actually is that the mesh being fine enough for the real NDF to be approximately uniform over each cell and coarse enough for having enough parcels in each cell. The collision substep then consists in applying a Monte-Carlo algorithm for computing in each cell an approximate solution of the spatially homogeneous Boltzmann equation:

$$
\frac{\partial f_{p}}{\partial t}\left(\mathbf{c}_{p} ; \mathbf{x}, t\right)=Q_{\text {coll }}\left\{f_{p}\right\}\left(\mathbf{c}_{p} ; \mathbf{x}, t\right)
$$

assuming that the initial NDF can be approximated by (20). It is worth noticing that the expression of $Q_{\text {coll }}$ in Eq. (21) relies on a closure assumption for the pair NDF which is usually based on the molecular chaos assumption.

\section{Extension to turbulent particle-laden flows}

In turbulent particle-laden flows, the fluid turbulent velocity is a new variable which has to be taken into account. In the framework of Monte-Carlo algorithms the approximation of the joint fluid-particle NDF by a linear combination of Dirac masses reads

$f_{f p}\left(\mathbf{c}_{p}, \mathbf{c}_{f} ; \mathbf{x}, t\right)=\sum_{i=1}^{N_{p a r}(t)} w_{p}^{i} \delta\left[\mathbf{x}-\mathbf{x}_{p}^{i}\right] \times \delta\left[\mathbf{c}_{p}-\mathbf{u}_{p}^{i}\right] \times \delta\left[\mathbf{c}_{f}-\mathbf{u}_{f @ p}^{i}\right]$

It can be noticed that in the literature this approximation is generally not explicitly written. The transport substep of the Monte-Carlo algorithm is supplemented by the prediction of the fluid velocity seen by the parcel (see Appendix B). In the collision substep the joint fluid-particle NDF, Eq. (22), is replaced by

$f_{f p}\left(\mathbf{c}_{p}, \mathbf{c}_{f} ; \mathbf{x}, t\right)=\sum_{C_{i}} \sum_{k \in \operatorname{Ind}\left(C_{i}\right)} w_{p}^{k} \frac{\mathbf{1}_{C_{i}}(\mathbf{x})}{\operatorname{vol}\left(C_{i}\right)} \delta\left[\mathbf{c}_{p}-\mathbf{u}_{p}^{k}\right] \times \delta\left[\mathbf{c}_{f}-\mathbf{u}_{f @ p}^{k}\right]$
Then the collision substep reduces to solve:

$\frac{\partial f_{f p}}{\partial t}\left(\mathbf{c}_{p}, \mathbf{c}_{f} ; \mathbf{x}, t\right)=Q_{\text {coll }}\left\{f_{f p}\right\}\left(\mathbf{c}_{p}, \mathbf{c}_{f} ; \mathbf{x}, t\right)$,

assuming that the initial joint fluid-particle NDF can be approximated by Eq. (23). Initially, people implicitly used the classical molecular chaos assumption to close the collision kernel (O'Rourke, 1981; Villedieu and Hylkema, 1997):

$f_{f p}^{(2)}\left(\mathbf{c}_{p}, \mathbf{c}_{f @ p}, \mathbf{c}_{q}, \mathbf{c}_{f @ q}\right)=f_{f p}\left(\mathbf{c}_{p}, \mathbf{c}_{f @ p}\right) f_{f p}\left(\mathbf{c}_{q}, \mathbf{c}_{f @ q}\right)$

and simply applied the standard Monte-Carlo algorithms formerly developed for the rarefied gas Boltzmann equation. As pointed out in the introduction, and highlighted in the following of the paper, this assumption leads to spurious effects due to the correlation between colliding particle velocities. To try to overcome this issue several attempts can be found in the literature (Sommerfeld, 1999; Berlemont et al., 2001) but are not fully satisfactory as explained in the introduction.

New collision algorithm in the framework of the joint fluid-particle NDF approach

The new proposed Monte-Carlo algorithm is based on the discretization of the collision kernel given by Eq. (16). In this expression, from a mathematical point of view, the $c_{f}$ variable plays a similar role as the space variable since, according to Eq. (16), collision may only occur between two particles which are located at the same location in the $\mathbf{c}_{f}$-space. Consequently, the approximation given by Eq. (23) is not suited for computing collision probabilities and must be replaced by another expression involving a continuous dependency with respect to the variable $\mathbf{c}_{f}$. Indeed, formally according to Eq. (23) two given particles either see the same fluid velocity and have the same velocity (when they belong to the same parcel) or see two different fluid velocities (when they belong to two different parcels). For this reason, Eq. (23) is clearly not compatible with the closure model (16) for the joint fluid-particle pair NDF. Whatever the stochastic algorithm used for simulating collisions, a $\mathbf{c}_{f}$-space regularization of Eq. (23) is needed in order to be able to compute pair collision probabilities at any given location in $\mathbf{c}_{f}$-space.

A natural solution consists in introducing a mesh $\mathcal{M}_{c_{f}}$ of the $\mathbf{c}_{f}$-space and to replace Eq. (23) by the following expression:

$f_{f p}\left(\mathbf{c}_{p}, \mathbf{c}_{f} ; \mathbf{x}, t\right)=\sum_{C_{i} \in \mathcal{M}_{x} Q_{j} \in \mathcal{M}_{c_{f}}} \sum_{k \in \operatorname{Ind}\left(C_{i}, Q_{j}\right)} w_{p}^{k} \frac{\mathbf{1}_{Q_{j}}\left(\mathbf{c}_{f}\right) \mathbf{1}_{c_{i}}(\mathbf{x})}{\operatorname{vol}\left(C_{i}\right) \operatorname{vol}\left(Q_{j}\right)} \delta\left[\mathbf{c}_{p}-\mathbf{u}_{p}^{k}\right]$

where $Q_{j}$ denotes a given cell of the mesh $\mathcal{M}_{c_{f}}, \operatorname{Ind}\left(C_{i}, Q_{j}\right)$ is the list of parcels located in $C_{i} \cap Q_{j}$ at the end of the transport step and $\operatorname{vol}\left(Q_{j}\right)$ is the volume of $Q_{j}$ in the $\mathbf{c}_{f}$-space. The corresponding Monte-Carlo collision algorithm then consists in the following steps:

(i) At the end of the transport step, for each cell $C_{i} \in \mathcal{M}_{x}$ and each cell $Q_{j} \in \mathcal{M}_{c_{f}}$, create the list $\operatorname{Ind}\left(C_{i}, Q_{j}\right)$ of the parcels located in both $C_{i}$ and $Q_{j}$.

(ii) Let $N_{i j}$ be the number of parcels in $\operatorname{Ind}\left(C_{i}, Q_{j}\right)$. Choose randomly $N_{i j} / 2$ pairs of potential collision partners or $\left(N_{i j}-1\right) / 2$ if $N_{i j}$ is odd and is larger than 2 .

(iii) For each pair, compute the collision probability:

$$
\begin{aligned}
& P_{\text {coll }}^{21}=w_{p 1} \frac{\left(N_{i j}-1\right)}{\operatorname{vol}\left(C_{i}\right)} \frac{\pi\left(d_{p 1}+d_{p 2}\right)^{2}}{4 P_{f j}}\left|\mathbf{c}_{p 2}-\mathbf{c}_{p 1}\right| \Delta t \\
& P_{\text {coll }}^{12}=w_{p 2} \frac{\left(N_{i j}-1\right)}{\operatorname{vol}\left(C_{i}\right)} \frac{\pi\left(d_{p 1}+d_{p 2}\right)^{2}}{4 P_{f j}}\left|\mathbf{c}_{p 2}-\mathbf{c}_{p 1}\right| \Delta t
\end{aligned}
$$


where $p 1$ and $p 2$ denote the corresponding parcels of the selected pair, $\Delta t$ is the time step. $P_{\text {coll }}^{21}$ denotes the probability for a given particle of parcel $p 2$ to collide with any particle of parcel $p 1$ during the current time step. In a similar manner $P_{\text {coll }}^{12}$ denotes the probability for a given particle of parcel $p 1$ to collide with any particle of parcel $p 2 . P_{f j}$ represents the probability for the fluid velocity to belong to $Q_{j}$ :

$P_{f j}=\int_{Q_{j}} P_{f}\left(\mathbf{c}_{f}\right) d \mathbf{c}_{f} \simeq \operatorname{vol}\left(Q_{j}\right) P_{f}\left(\mathbf{c}_{f_{j}}\right)$

where $\mathbf{c}_{f_{j}}$ is the centre of $Q_{j}$.

(iv) Choose a random number $\alpha$ between 0 and 1 . If $\alpha \leqslant P_{\text {coll }}^{21}$, then all the particles of parcel $p 2$ are supposed to have actually collided with a particle of parcel $p 1$ during the time step. Then parcel $p 2$ new velocity is updated according to Eq. (8). If $\alpha \leqslant P_{\text {coll }}^{12}$, then all the particles of parcel $p 1$ are supposed to have actually collided with a particle of parcel $p 2$ during the time step. Then parcel $p 1$ new velocity is updated according to Eq. (7). In both cases the fluid velocities are left unchanged.

In case of coalescence, the algorithm is slightly modified as follows. Let $P_{\text {coll }}^{\max }=\max \left(P_{\text {coll }}^{12}, P_{\text {coll }}^{21}\right)$ then if $\alpha \leqslant P_{\text {coll }}^{\max }$, then the collision between $p 1$ and $p 2$ is supposed to be effective. Assuming that $w_{p 2}>w_{p 1}$ the new parcel characteristics are then defined as: $w_{p 2}^{*}=w_{p 2}-w_{p 1}$ with a mass and velocity given by and Eqs. (9) and (10) and the parcel $p 1$ is unchanged $w_{p 1}^{*}=w_{p 1}$.

It is worth noticing that, by construction, the above Monte-Carlo algorithm is a generalization of chaos assumption based algorithms used by O'Rourke (1981) and Villedieu and Hylkema (1997). Indeed if the mesh $\mathcal{M}_{c_{f}}$ only contains one cell corresponding to the whole velocity space, one has: $P_{f j}=1$ and one recovers the usual expression for the collision probability. To account for the correlations induced by the fluid velocity, it is necessary to use a mesh of the " $\mathbf{c}_{f}$-space" which is fine enough. This question will be quantified in the next section.

It is also worth pointing out that for the above algorithm to be consistent with the kinetic equation, it is mandatory that, whatever the particle pairing, the condition $P_{\text {coll }} \Delta t \leqslant 1$ be satisfied. This yields a restrictive condition on the time step, which reads:

$\Delta t \leqslant \operatorname{Min}_{i, j} \frac{P_{f j}}{\left(N_{i j}-1\right)} \frac{v o l\left(C_{i}\right)}{w_{\max } d_{\max }^{2} c_{\max }}$

where $c_{\max }$ denotes the maximal relative velocity between two parcels located in the same control volume $C_{i} \cap Q_{j}, w_{\max }$ the maximum weight of the parcels and $d_{\max }$ denotes the largest particle diameter. Since the fluid velocity density function $P_{f}$ is not explicitly known in practice, $P_{f j}$ may be approximated by:

$P_{f j}=\frac{N_{i j}}{N_{i}}$

with $N_{i}=\sum_{j} N_{i j}$. Inserting Eq. (31) in Eq. (30) finally yields the following constraint on the time step:

$\Delta t \leqslant \operatorname{Min}_{i, j} \frac{N_{i j}}{\left(N_{i j}-1\right)} \frac{\operatorname{vol}\left(C_{i}\right)}{w_{\max } N_{i} d_{\max }^{2} c_{\max }}$

which physically means that the time step must be lower than the characteristic collision time scale.

Actually, the constraint on the time step does not prevent to use a large number of parcels in a cell. This is due to the fact that, for a given fixed number of real particles in the computational domain, if one lets the number of parcels per cell $N_{i}$ going to infinity, then the corresponding numerical weight of each parcel $w_{p}$ will tend to 0 while $N_{i} \times w_{p}$ will remain constant. If, for the sake of simplicity, one assumes that all parcels have the same weight $w$ and that all particles have the same diameter $d$, the constraint (32) can be simplified and replaced by the sufficient condition:

$\Delta t \leqslant \frac{\operatorname{vol}\left(C_{i}\right)}{N_{i} w d^{2} c_{\max }}$

It is worth noticing that $N_{i} w / \operatorname{vol}\left(C_{i}\right)$ actually represents the real particle density in cell $C_{i}$. Hence, the right-hand side of Eq. (33) actually corresponds to the shortest collision time between two real particles located in cell $C_{i}$. The constraint given by Eq. (32) has thus a clear physical meaning and just expresses that the time step must not be larger than the real collision time scale.

If all parcels have the same weight, it is worth noticing that, according step (iv) of the collision algorithm, parcel $p 1$ and parcel p2 always collide simultaneously. Consequently momentum and particle kinetic energy (in case of elastic collisions) are exactly conserved during the collision step. If the weights are not the same, conservation is not ensured exactly but is only satisfied in a statistical sense.

The presence of the term $\left(N_{i j}-1\right)$ in the expression of the collision probability can be easily explained. Indeed, the total number of pairs that could be formed with $N_{i j}$ parcels is equal to $\left(N_{i j}-1\right) N_{i j} / 2$. Given that, according to the collision algorithm, only $N_{i j} / 2$ pairs are considered at each time step meaning that the probability of finding a given pair is underestimated by a factor of $\left(N_{i j}-1\right)$ which has thus to be taken into account to get the correct value of the collision probability.

In the following sections our new proposed algorithm is called shortly "CCV Monte-Carlo algorithm" for Correlated Colliding Velocity Monte-Carlo algorithm.

\section{Effect of the Monte-Carlo algorithm's parameters}

As described in the previous section, the proposed Monte-Carlo algorithm introduces several additional parameters than a standard Monte-Carlo algorithm. For quantifying the sensitivity of the Monte-Carlo algorithm to these parameters many numerical tests have been conducted. In this section all numerical simulations have been performed with the parameters of the turbulence given in Table 1 . The particles are spherical, monodisperse and elastic $\left(e_{c}=1\right)$ and their material properties have been chosen for having an important effect of the turbulence on the collision.

Table 1

Gas and particle material properties for DNS of colliding particles.

\begin{tabular}{|c|c|c|c|c|c|c|}
\hline \multicolumn{7}{|l|}{ Fluid properties } \\
\hline \multicolumn{5}{|l|}{ Reynolds number, $R e$} & \multicolumn{2}{|l|}{104} \\
\hline \multirow{2}{*}{\multicolumn{5}{|c|}{$\begin{array}{l}\text { Reynolds number, } R e_{\lambda} \\
\text { Viscosity, } \mu_{f}(\mathrm{~kg} / \mathrm{m} / \mathrm{s})\end{array}$}} & \multicolumn{2}{|l|}{50.3} \\
\hline & & & & & \multicolumn{2}{|c|}{$1.72 \times 10^{-5}$} \\
\hline \multicolumn{5}{|l|}{ Density, $\rho_{f}\left(\mathrm{~kg} / \mathrm{m}^{3}\right)$} & \multicolumn{2}{|c|}{1.17} \\
\hline \multicolumn{5}{|c|}{ Turbulence kinetic agitation, $q_{f}^{2}\left(\mathrm{~m}^{2} / \mathrm{s}^{2}\right)$} & \multicolumn{2}{|c|}{$30.1 \times 10^{-3}$} \\
\hline \multicolumn{4}{|l|}{ Turbulent time scale, $\tau_{f}^{t}(\mathrm{~s})$} & & \multicolumn{2}{|c|}{$61.2 \times 10^{-3}$} \\
\hline Symbols & $\square$ & $\triangle$ & $\nabla$ & $\triangleright$ & $\triangleleft$ & $\diamond$ \\
\hline \multicolumn{7}{|l|}{ Particle properties } \\
\hline Diameter, $d_{p}(\mu \mathrm{m})$ & 600 & & & & & \\
\hline Density, $\rho_{p}\left(\mathrm{~kg} / \mathrm{m}^{3}\right)$ & 18.75 & 37.50 & 75.00 & 150.0 & 300.0 & 450.0 \\
\hline Stokes number, $\tau_{f p}^{F} / \tau_{f @ p}^{t}$ & 0.22 & 0.43 & 0.85 & 1.70 & 3.22 & 4.58 \\
\hline $\begin{array}{l}\text { Lagrangian time scale, } \\
\quad \tau_{f @ p}^{t} / \tau_{f}^{t}\end{array}$ & 1.23 & 1.20 & 1.11 & 1.06 & 1.09 & 1.13 \\
\hline $\begin{array}{l}\text { Fluid turbulent kinetic } \\
\quad \text { energy, } q_{f @ p}^{2} / q_{f}^{2}\end{array}$ & 0.96 & 0.94 & 0.96 & 0.97 & 0.99 & 1.00 \\
\hline Restitution coefficient, $e_{c}$ & 1.0 & & & & & \\
\hline
\end{tabular}


So the particles are the ones of Table 1 with $\rho_{p}=75 \mathrm{~kg} / \mathrm{m}^{3}$. Each numerical simulation has been performed as follows. First a transitory phase is performed of approximately $50 \tau_{f}^{t}$ allowing the fluid and particles velocities to reach a statistically steady state (initial fluid and particle velocities being zero). Then statistics are computed also during $50 \tau_{f}^{t}$. The numerical tests have shown that the relevant parameters of the CCV Monte-Carlo algorithm are (i) the number of particles in each section of discretization of $c_{f}$, (ii) the range of the fluid realization considered for the discretization, and (iii) the size of the section (namely $\Delta c_{f}$ ).

Figs. 1 and 2 show the particle kinetic energy and the collision time scale with respect to $N_{p a r} / N_{s e c}^{3}$ which is the number of parcel

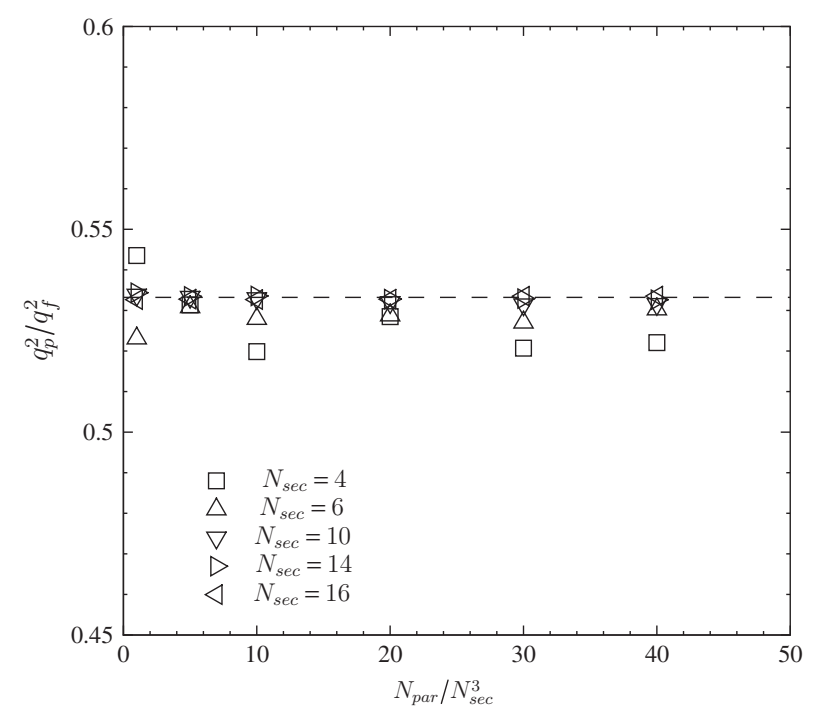

Fig. 1. Dependence of the CCV Monte-Carlo algorithm predictions of the kinetic energy of particles suspended in homogeneous isotropic gas turbulence on the number of parcels per section $N_{\text {par }} / N_{\text {sec }}^{3}$. The computations were performed with several number of uniform sections for discretizing the gas velocity space ( $c_{f}$-space) in a region defined from $-\max \left(\left|c_{f}\right|\right)$ to $+\max \left(\left|c_{f}\right|\right)$ with $\max \left(\left|c_{f}\right|\right)=3 \times \sqrt{2 / 3 q_{f}^{2}}$.

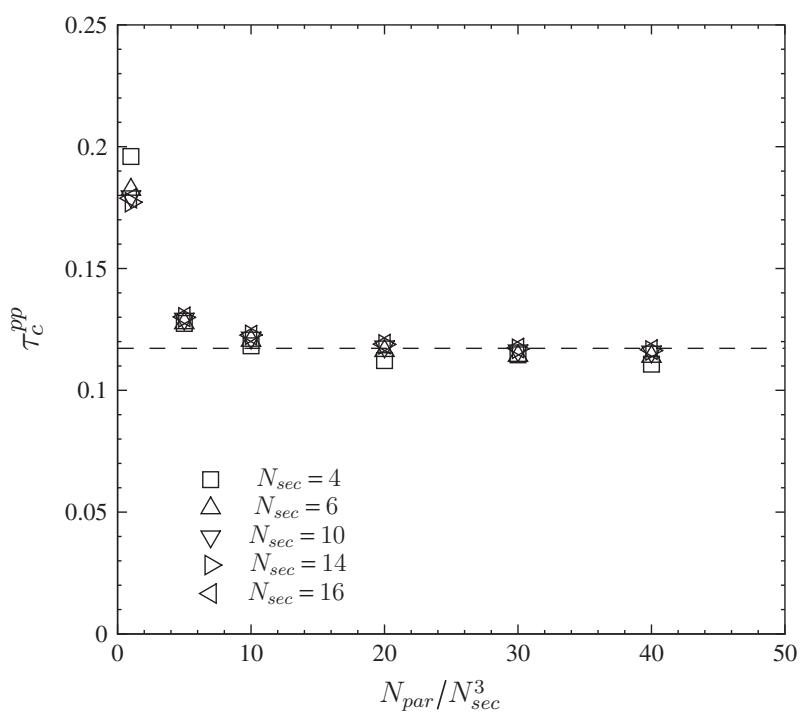

Fig. 2. Dependence of the CCV Monte-Carlo algorithm predictions of the interparticle collision time scale of particles suspended in homogeneous isotropic gas turbulence on the number of parcels per section $N_{p a r} / N_{s e c}^{3}$. The computations were performed with several number of uniform sections for discretizing the gas velocity space $\left(c_{f}\right.$-space) in a region defined from $-\max \left(\left|c_{f}\right|\right)$ to $+\max \left(\left|c_{f}\right|\right)$ with $\max \left(\left|c_{f}\right|\right)=3 \times \sqrt{2 / 3 q_{f}^{2}}$. per section for a uniform distribution of $c_{f}$. Obviously, due to the Gaussian distribution of fluid velocity realization, $N_{p a r} / N_{s e c}^{3}$ is not strictly the number of particles per section. However, it is an independent parameter characterizing the stochastic collision algorithm. Fig. 1 shows that the particle kinetic energy converges very quickly to a value independent of $N_{p a r} / N_{s e c}^{3}$. The discrepancies observed for $N_{s e c}=4$ and $N_{s e c}=6$ come from the fact that such number of section lead to a small number of particles. An averaging over a period longer than $50 \tau_{f}^{t}$ should remove the discrepancies. The collision time scale, shown by Fig. 4, is more influenced by the number of particle per section. The convergence is obtained for approximately $N_{\text {par }} / N_{\text {sec }}^{3}=12$.

The effect of the range of the fluid velocity realization space is shown by Figs. 3 and 4 . In these numerical simulations the number of sections is kept constant $\left(N_{\text {sec }}=10\right)$ leading to non-constant $\Delta c_{f}$ from one value of $\max \left(\left|c_{f}\right|\right)$ to another one. Fig. 3 shows that the particle kinetic energy is nearly constant for small values of $\max \left(\left|c_{f}\right|\right) / \sqrt{2 / 3 q_{f}^{2}}$. This trend was expected because in such a case, the number of parcels allowed to collide is reduced and consequently the destruction of particle kinetic energy by collision is reduced. An interesting point is for increasing the fluid velocity realization space the particle kinetic energy is found decreasing. As more detailed hereafter, it comes from the fact that for increasing $\Delta c_{f}$ the span of the fluid velocities in a section is increasing respectively leading to an increase of the particle kinetic energy destruction. As expected, for a small range of fluid velocity realization $\max \left(\left|c_{f}\right|\right)=\sqrt{2 / 3 q_{f}^{2}}$ the collision time scale is overestimated always because the number of parcels having a fluid velocity between $-\sqrt{2 / 3 q_{f}^{2}}$ and $\sqrt{2 / 3 q_{f}^{2}}$ is too small. Fig. 4 shows that for $\max \left(\left|c_{f}\right|\right) \geqslant 2 \times \sqrt{2 / 3 q_{f}^{2}}$ the inter-particle collision time scale converged as soon as the number of parcel per section is $N_{p a r} / N_{s e c}^{3}>5$.

The effect of the mesh size of the fluid velocity realization is shown by Figs. 5 and 6 . As already emphasized a large size of fluid velocity section means that the particles sorted inside are viewing a large distribution of fluid velocity. Consequently the destruction

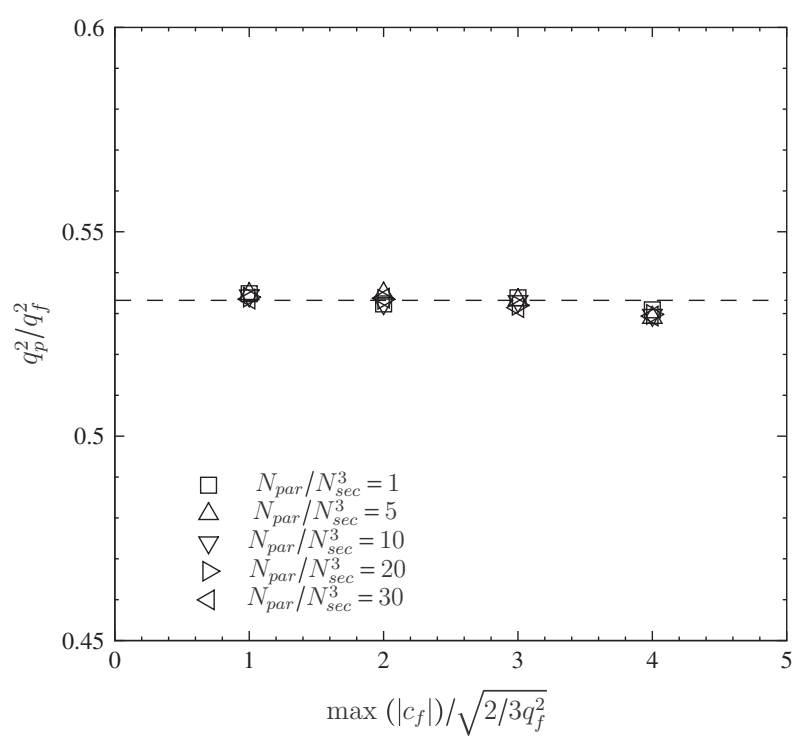

Fig. 3. Dependence of the CCV Monte-Carlo algorithm predictions of particles suspended in homogeneous isotropic gas turbulence on the size of the discretized region in the gas velocity space $\left(c_{f}\right.$-space). The computations were performed with 10 equal sections for discretizing the gas velocity space $\left(c_{f}\right.$-space) from $-\max \left(\left|c_{f}\right|\right)$ to $+\max \left(\left|c_{f}\right|\right)$. 


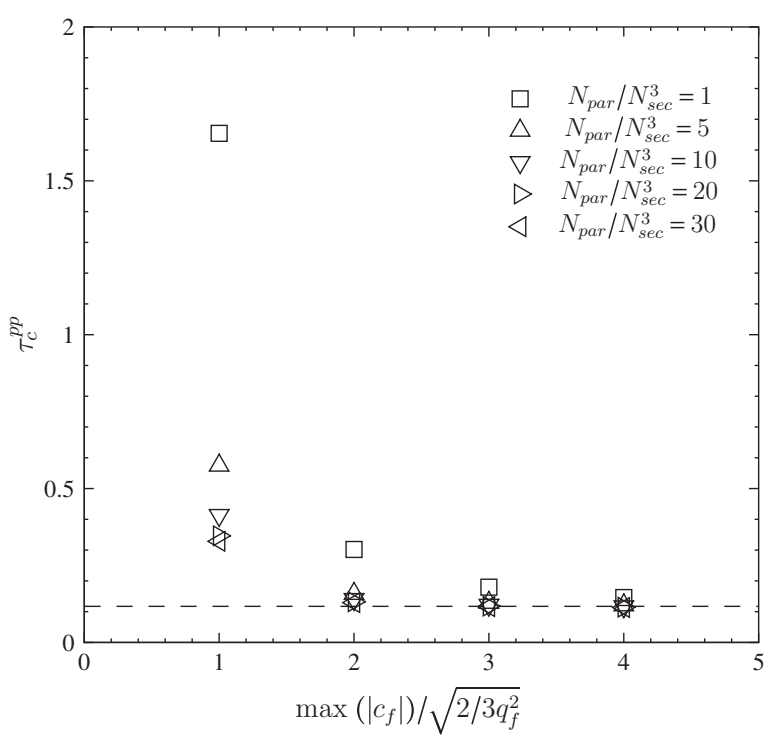

Fig. 4. Dependence of the CCV Monte-Carlo algorithm predictions of the interparticle collision time scale of particles suspended in homogeneous isotropic gas turbulence on the size of the discretized region in the gas velocity space ( $c_{f}$-space). The computations were performed with 10 equal sections for discretizing the gas velocity space $\left(c_{f}\right.$-space) from $-\max \left(\left|c_{f}\right|\right)$ to $+\max \left(\left|c_{f}\right|\right)$.

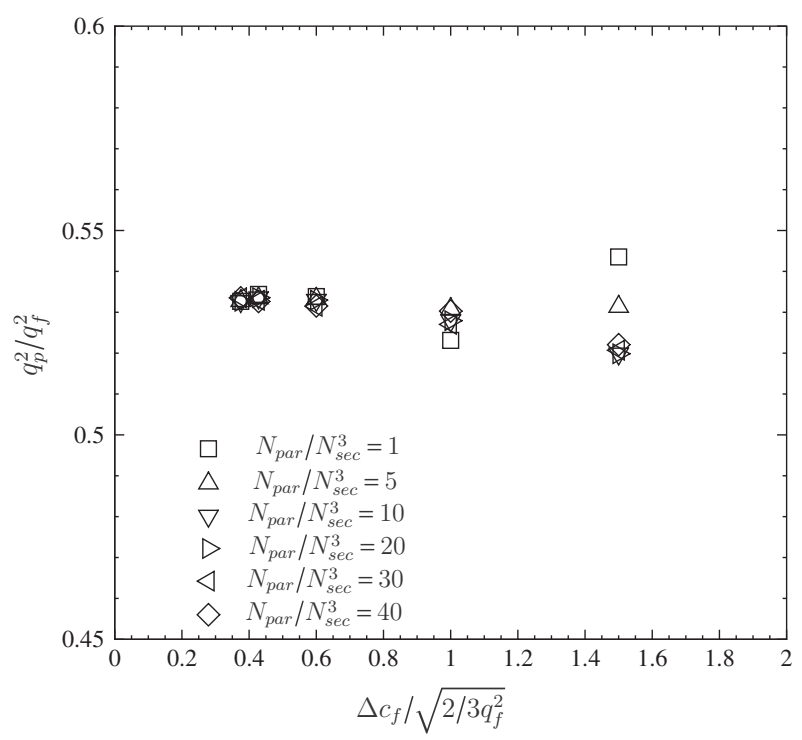

Fig. 5. Dependence of the CCV Monte-Carlo algorithm predictions of the kinetic energy of particles suspended in homogeneous isotropic gas turbulence on the size of the mesh used to discretize the gas velocity space ( $c_{f}$-space). The computations were performed with several number of uniform sections for discretizing the gas velocity space $\left(c_{f}\right.$-space) in a region defined from $-\max \left(\left|c_{f}\right|\right)$ to $+\max \left(\left|c_{f}\right|\right)$ and with $\max \left(\left|c_{f}\right|\right)=3 \times \sqrt{2 / 3 q_{f}^{2}}$.

of the particle kinetic energy is enhanced. Indeed, Fig. 5 shows that for $\Delta c_{f}=1.5 \times \sqrt{2 / 3 q_{f}^{2}}$ the particle kinetic energy decreases even for a large number of parcel per section. In contrast, for very small mesh size the particle kinetic energy is conserved by the CCV algorithm. However, the reader must be aware that for very small $\Delta c_{f}$ and for a small number of parcel the number of parcel per section becomes too small for predicting the true collision frequency. Fig. 6 clearly shows this trend because for $N_{\text {par }} / N_{\text {sec }}^{3}=1$ the inter-particle collision time scale is overestimated meaning that just a few number of collisions are predicted by the Monte-Carlo algorithm.

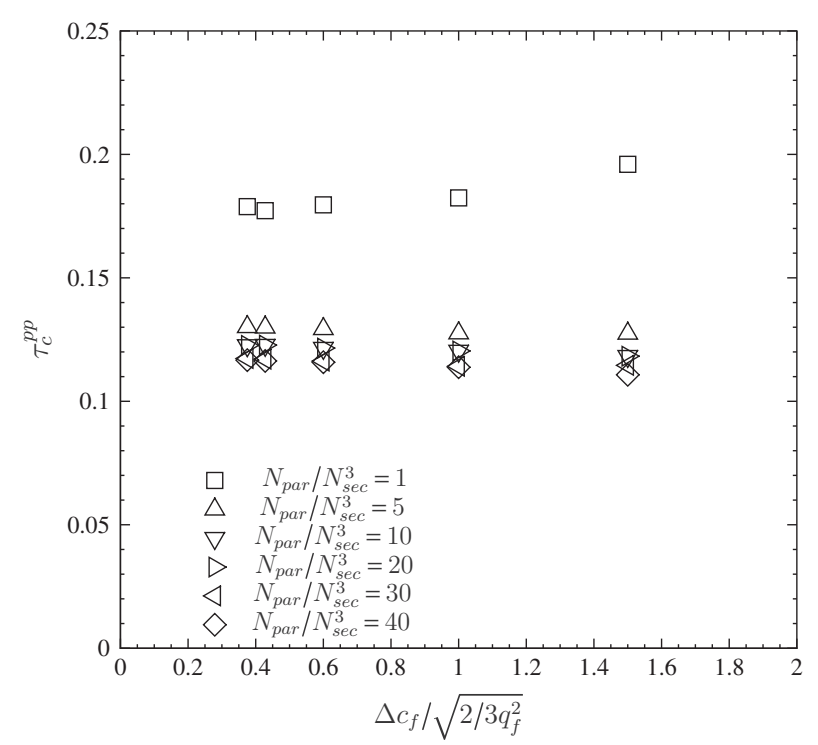

Fig. 6. Dependence of the CCV Monte-Carlo algorithm predictions of the interparticle collision time scale of particles suspended in homogeneous isotropic gas turbulence on the size of the mesh used to discretize the gas velocity space $\left(c_{f}\right.$ space). The computations were performed with several number of uniform sections for discretizing the gas velocity space $\left(c_{f}\right.$-space) in a region defined from $-\max \left(\left|c_{f}\right|\right)$ to $+\max \left(\left|c_{f}\right|\right)$ and with $\max \left(\left|c_{f}\right|\right)=3 \times \sqrt{2 / 3 q_{f}^{2}}$.

To summarize, the important parameters of the CCV Monte-Carlo algorithm are: the number of parcel in a section $N_{\text {par }} / N_{\text {sec }}^{3}$, the maximum of the range of the meshed fluid velocity space realizations $\max \left(\left|c_{f}\right|\right)$ and finally the size of the mesh $\Delta c_{f}$. Even if it could be surprising the number of section is a consequence of these three parameters. Typically, the numerical parameters can be chosen as $\max \left(\left|c_{f}\right|\right)=3 \times \sqrt{2 / 3 q_{f}^{2}}$ and $\Delta c_{f}=0.5 \times$ $\sqrt{2 / 3 q_{f}^{2}}$. The number of sections is $N_{\text {sec }}=2 \times \max \left(\left|c_{f}\right|\right) / \Delta c_{f}=$ $2 \times 3 / 0.5=12$ that leads to $12^{3} c_{f}$-space cells. For $N_{p a r} / N_{s e c}^{3}=10$, the number of parcels becomes $N_{p a r}=17,280$.

In the following sections the predictions of the CCV Monte-Carlo algorithm is compared with deterministic simulation results (DPS/DNS or DPS/LES), and with analytical results from moment approach. To emphasize the benefit of the CCV Monte-Carlo algorithm the prediction of the standard Monte-Carlo algorithm, namely based on the molecular chaos assumption, are also shown.

\section{Monodisperse solid particles}

\section{Description of the numerical simulations}

In this section the Monte-Carlo algorithm is applied to the numerical simulation of solid colliding particles suspended in statistically steady homogeneous isotropic turbulent flow. The computational domain is a cubic box of length $L_{b}=0.128 \mathrm{~m}$ with full periodic boundary conditions. All relevant physical properties are gathered in Table 1.

This configuration of an homogeneous isotropic flow with colliding particles has been extensively investigated by DPS/DNS or DPS/LES (Deutsch and Simonin, 1991; Laviéville et al., 1997; Février et al., 2005; Fede and Simonin, 2006; Wunsch et al. 2008; Fede and Simonin, 2010). The methods and the equations solved are briefly given in Appendix A. Fig. 7 shows the particle kinetic energy, normalized by the fluid turbulent kinetic energy, with respect to the inverse of the Stokes number. As expected, 


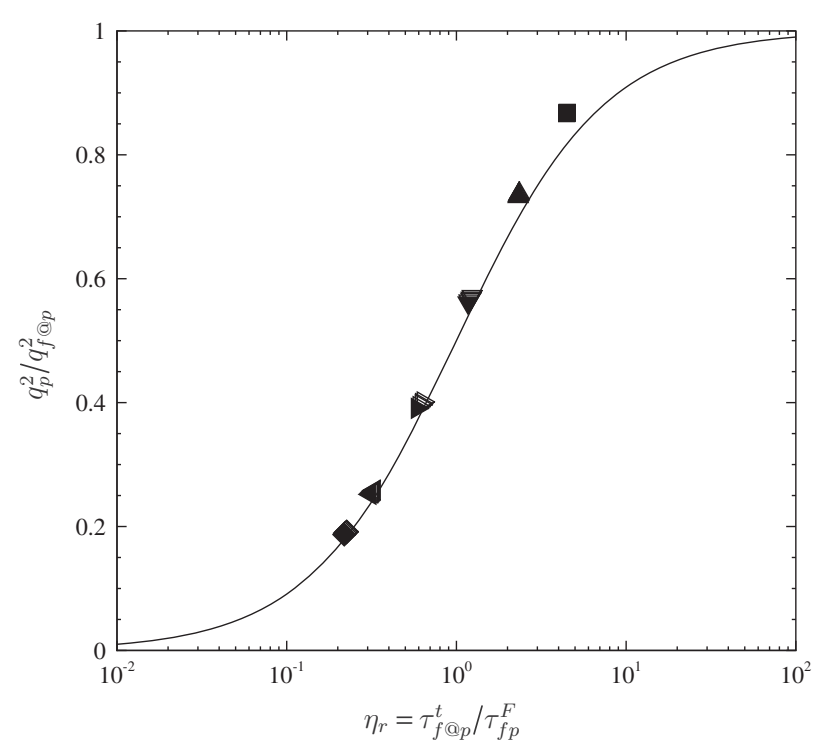

Fig. 7. Dependence of the particle kinetic energy (normalized by the fluid turbulent kinetic energy seen by the particle) on the inverse of the Stokes number measured in deterministic simulation DPS/DNS. The black-filled symbols correspond to the case without collisions, the empty symbols with collisions, and the solid line is the moment method given by Eq. (38).

the figure shows that the particle kinetic energy is conserved for all considered solid volume fractions because the collisions are elastic $\left(e_{c}=1\right)$. Moreover, the particle kinetic energy is the same than in the case without collisions (the black-filled symbols).

\section{Monodisperse elastic solid particles}

The dispersed phase is composed of $N_{p}$ identical solid spheres. The particle velocities after a collision are computed with Eqs. (7) and (8) corresponding to a pure sliding collision. It is important to notice that the numerical simulations were carried out for elastic particles therefore the particle restitution coefficient, $e_{c}$, is equal to 1 . The consequence is that the collisions conserve the particle kinetic energy.

The solid lines shown by Fig. 7 are the predictions of the moment method (Simonin, 1996). From the Boltzmann-like equation (1) it is possible to derive the transport equation of any moment of the joint fluid-particle NDF especially for the particle kinetic energy $\left.\left.q_{p}^{2}=1 / 2\langle u\rangle_{p, i} u\right\rangle_{p, i}\right\rangle$, and the fluid-particle velocity covariance $q_{f p}=\left\langle u_{f @ p, i} u u_{p, i}\right\rangle$. In homogeneous particle-laden turbulent flows, these transport equations write,

$$
\begin{aligned}
& \frac{d q_{p}^{2}}{d t}=-\frac{2 q_{p}^{2}-q_{f p}}{\tau_{f p}^{F}}+\mathbb{C}\left(q_{p}^{2}\right) \\
& \frac{d q_{f p}}{d t}=-\frac{q_{f p}-2 q_{f @ p}^{2}}{\tau_{f p}^{F}}-\frac{q_{f p}}{\tau_{f @ p}^{t}}+\mathbb{C}\left(q_{f p}\right)
\end{aligned}
$$

where the two last terms on the right-hand side represent the effects of the collisions. The modification of fluid-particle velocity covariance by a collision can be determined from Eqs. (7) and (8) using $\Delta\left[q_{f p}\right]=-1 / 2\left(\mathbf{u}_{f @ q}-\mathbf{u}_{f @ p}\right) \cdot \mathbf{w}_{p q}$. This expression, obviously shows that if the fluid velocity seen by colliding particles is same then $\mathbf{u}_{f @ q}=\mathbf{u}_{f @ p}$, the modification of the fluid-particle velocity covariance by a collision vanishes, and then $\mathbb{C}\left(q_{f p}\right)=0$. Assuming a Maxwellian distribution function $f_{p}$ and molecular chaos the collision term writes

$\mathbb{C}\left(q_{p}^{2}\right)=-\frac{1-e_{c}^{2}}{3 \tau_{k}^{p p}} \frac{2}{3} q_{p}^{2}$ where $\tau_{k}^{p p}$ is the particle-particle collision time scale derived in the framework of the kinetic theory of dilute gases of rigid elastic spheres (Chapman and Cowling, 1970),

$\frac{1}{\tau_{k}^{p p}}=4 \pi n_{p} d_{p}^{2} \sqrt{\frac{2}{3 \pi} q_{p}^{2}}$.

In the case of very dilute flow where the collisions can be neglected, or for elastic particles $e_{c}=1$, the modification of particle kinetic energy by particle-particle interaction becomes $\mathbb{C}\left(q_{p}^{2}\right)=0$ and assuming a steady flow, Eqs. (34) and (35) become

$2 q_{p}^{2}=q_{f p}=2 q_{f @ p}^{2} \frac{\eta_{r}}{1+\eta_{r}}$

where $\eta_{r}=\tau_{f @ p}^{t} / \tau_{f p}^{F}$. Fig. 7 shows that, independently of the solid volume fraction, the results from DPS/DNS are in accordance with the predictions of Eq. (38). In contrast, Fig. 8 exhibits that when the collision frequency is increasing, the particle kinetic energy is decreasing whereas the particles are elastic $\left(e_{c}=1\right)$. Laviéville et al. $(1995,1997)$ attribute such a pathological behaviour to decorrelation effect between the particle and the fluid turbulence due to the molecular chaos assumption used to compute the collision probability. Indeed, using the molecular chaos assumption for the pair joint fluid-particle NDF leads to Eq. (25). One can notice that such an approximation is worse than assuming molecular chaos only for the pair particle velocity $f_{p}^{(2)}$ distribution function (Eq. (14)). Indeed, in Eq. (25) it is assumed both that (i) the colliding particle velocities are uncorrelated and (ii) that the fluid velocity seen by the colliding particles are also uncorrelated. Point (i) can be legitimate in case of heavy particles, but (ii) can be true only for two particles having a distance larger than the turbulent length macro-scale. However Eq. (25) is used for colliding particles so that the inter-particle distance is equal to the particle diameter. Using the molecular chaos assumption for computing $\mathbb{C}\left(q_{f p}\right)$ leads to

$\mathbb{C}\left(q_{f p}\right)=-\frac{1+e_{c}}{3 \tau_{k}^{p p}} q_{f p}$

with $\tau_{k}^{p p}$ given by Eq. (37). Such a term is a destruction of the fluidparticle velocity covariance by collision. Fig. 9 shows the fluid-

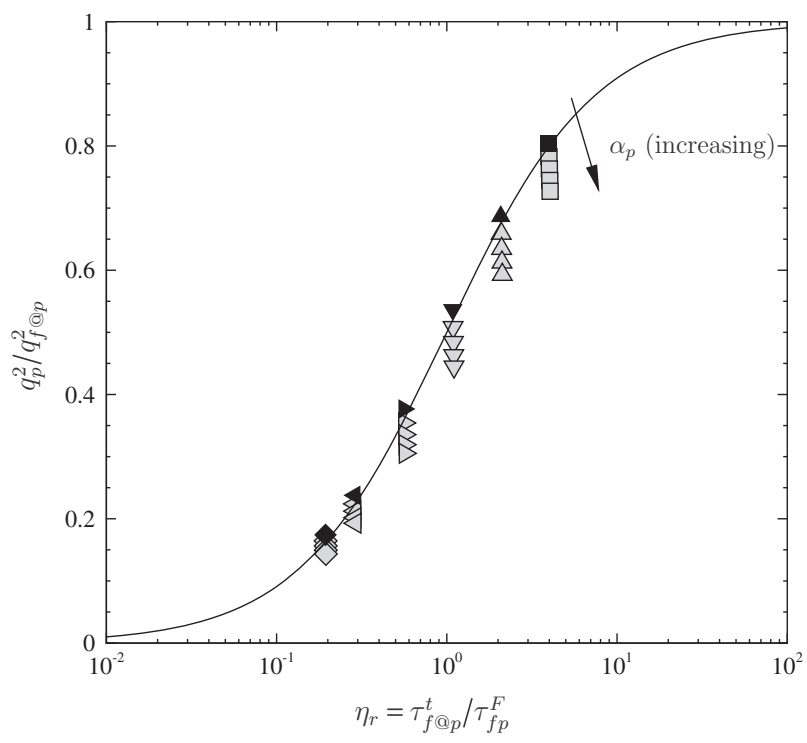

Fig. 8. Dependence of the particle kinetic energy (normalized by the fluid turbulent kinetic energy measured along the particle trajectories) on the inverse of the Stokes number in DPS/RANS using the standard Monte-Carlo algorithm (i.e. based on molecular chaos assumption). The black-filled symbols correspond to the case without collisions, the empty symbols with collisions, and the solid line is the moment method given by Eq. (38). 


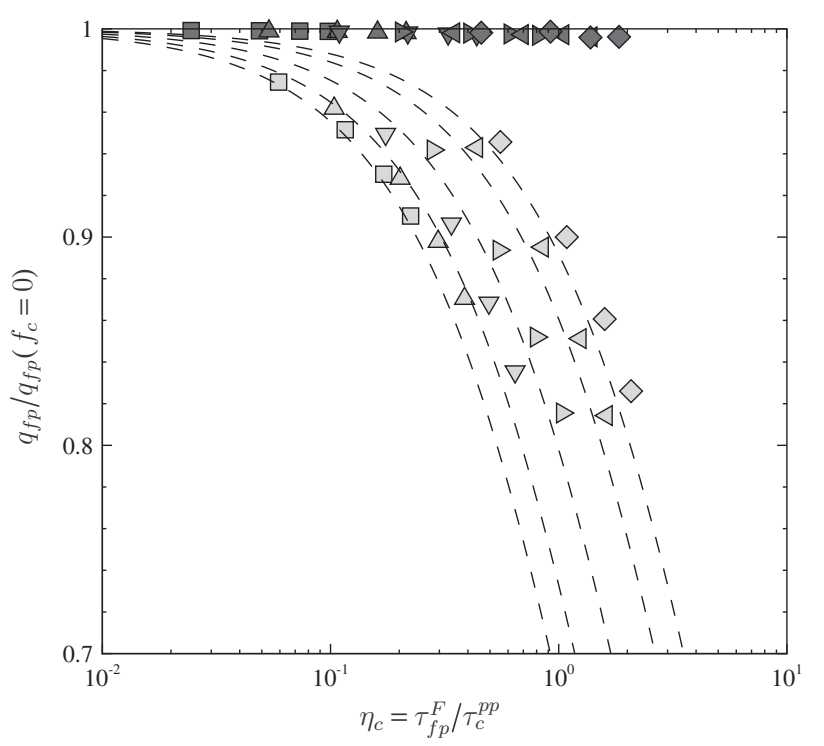

Fig. 9. Fluid-particle velocity covariance normalized by the value in case without collisions with respect to the collision frequency. The grey-filled symbols correspond to the results from the standard Monte-Carlo algorithm and black-filled symbols from the CCV Monte-Carlo algorithm. The dashed lines are the prediction of Eq. (40) for fluid-particle velocity covariance.

particle velocity covariance, normalized by the fluid-particle velocity covariance without collisions, with respect to the collision frequency. It is observed that for all considered Stokes numbers the fluid-particle velocity covariance is destroyed when the collision frequency is increasing. The fictitious destruction of the fluidparticle velocity covariance can be reproduced by the moment method (the solid lines) when the spurious destruction effect, given by Eq. (39), is taken into account:

$\frac{q_{f p}}{q_{f p}\left(f_{c}=0\right)}=\left[1+\frac{\eta_{r}}{1+\eta_{r}} \frac{2}{3} \eta_{c}\right]^{-1}$

where $\eta_{c}=\tau_{f p}^{F} / \tau_{c}^{p p}$. Fig. 9 shows also the fluid-particle velocity covariance from the numerical simulation performed with the CCV Monte-Carlo algorithm (the black-filled symbols). With CCV Monte-Carlo algorithm the fluid-particle velocity covariance is conserved because the equality $\mathbb{C}\left(q_{f p}\right)=0$ is accurately satisfied. For the largest values of the collision frequency a small destruction of the fluid-particle velocity covariance can be noticed. Fig. 10 shows that the CCV Monte-Carlo algorithm conserves the particle kinetic energy. Figs. 11 and 12 show the particle-particle collision time scale measured in the numerical simulation with respect to the Stokes number and the particle-particle mean relative velocity respectively. As expected the collision time scale predicted by the standard Monte-Carlo algorithm is in accordance with the prediction based on molecular chaos assumption Eq. (37). Laviéville et al. (1995) used the correlated colliding velocity closure for the pair joint fluid-particle NDF given by (16) and assumed a Maxwellian distribution of the joint fluid-particle distribution function to compute the following expression for the inter-particle collision time scale $\tau_{c}^{p p}$ :

$\tau_{c}^{p p}=\frac{\tau_{k}^{p p}}{\sqrt{1-\xi_{f p}^{2}}}$

where $\xi_{f p}=q_{f p} / 2 / \sqrt{q_{f @ p}^{2} q_{p}^{2}}$ is the fluid-particle velocity correlation coefficient $\xi_{f p} \in[0,1]$. Fig. 11 shows that the collision time scale measured in CCV algorithm is in accordance with Eq. (41). This trend was expected because the CCV algorithm and Eq. (41) are both based on the same model of the pair joint fluid-particle

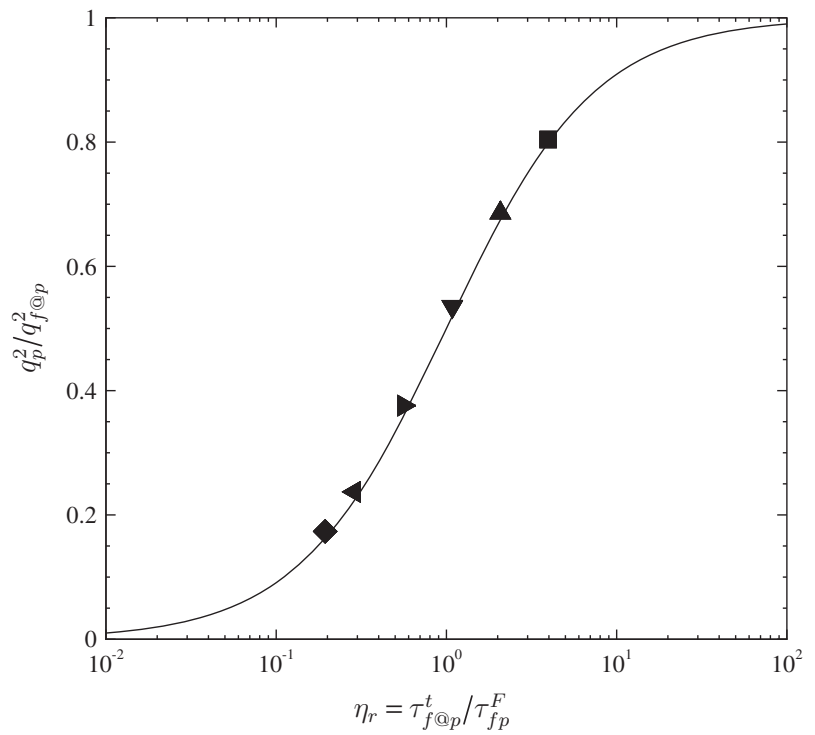

Fig. 10. Dependence of the particle kinetic energy (normalized by the fluid turbulent kinetic energy measured along the particle trajectories) on the inverse of the Stokes number. Results with CCV Monte-Carlo algorithm, namely based on correlated colliding velocities, with 16 sections. The black-filled symbols correspond to the case without collisions, the empty symbols with collisions, and the solid line is the moment method given by Eq. (38).

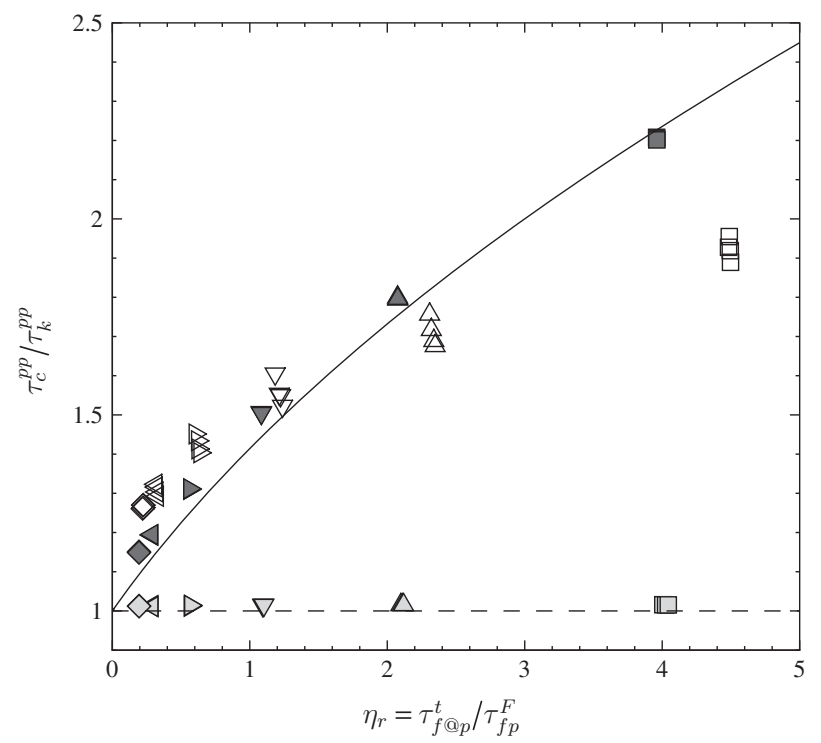

Fig. 11. Dependence of the inter-particle collision time scale normalized by the prediction of the kinetic theory Eq. (37) with respect to the inverse of the Stokes number. Empty symbols are the DPS/DNS, grey-filled symbols stochastic simulation with standard Monte-Carlo algorithm and the black-filled symbols with the CCV Monte-Carlo algorithm. The solid line is the prediction by Eq. (41).

NDF. The differences between DPS/DNS and DPS/RANS, shown by Fig. 11, come mainly from the fact that the preferential concentration effects are neglected in the closure of the joint fluid-particle NDF kinetic equation (given in Section 'Statistical description of binary collision between particles transported by a turbulent flows'). Indeed it has been shown that for solid particles having a response time of the order of the large turbulent scales, the particles may concentrate in low-vorticity regions of the turbulence (see Fig. 13) (Squires and Eaton, 1991; Fessler et al., 1994; Reade and Collins, 2000). On the one hand, as explained in Section 'Pair number distribution function closure', the closure of the pair NDF is done by assuming that the radial distribution function is equal to 1 


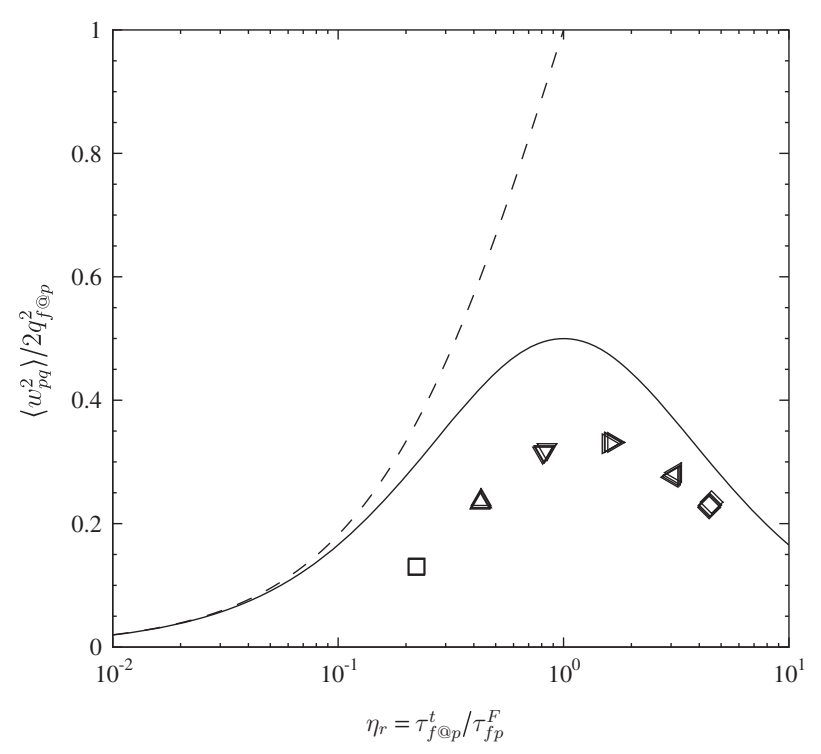

Fig. 12. Particle-particle relative velocity normalized by the fluid kinetic energy seen by the particles. The symbols are the DPS/DNS, the dashed lines the predictions based on the molecular assumption and the solid line the predictions with the correlated velocity closure.

(particles are uniformly randomly distributed). On the other hand, the fluid turbulent time scale and kinetic energy used in the Lagrangian stochastic process (B.1) are assumed to be independent of the Stokes number value $\left(\tau_{f @ p}^{t}=\tau_{f}^{t}\right.$ and $\left.q_{f @ p}^{2}=q_{f}^{2}\right)$. So it is important to notice that this is not an inherent limitation of the proposed Monte-Carlo algorithm and that the preferential concentration effects could be introduced though the modelling of the radial function $g_{0}$ and of the fluid turbulent time scale $\tau_{f @ p}^{t}$ by using, for example, a given function of the Stokes number or measured values from the corresponding DPS/DNS results.

\section{Monodisperse inelastic solid particles}

The present section scrutinizes the efficiency of the CCV Monte-Carlo algorithm for predicting the dynamical behaviour of non-elastic solid particles suspended in homogeneous isotropic turbulent flows. The properties of the turbulence are given by Table 1 . Only two kinds of particles are considered differing by their densities: $\rho_{p}=75 \mathrm{~kg} / \mathrm{m}^{3}(\nabla)$ and $\rho_{p}=300 \mathrm{~kg} / \mathrm{m}^{3}(\triangleleft)$ and for each density two solid volume fraction have been considered $\alpha_{p}=13.48 \times 10^{-3}$ and $\alpha_{p}=53.93 \times 10^{-3}$. The normal restitution coefficient has been taken such as $e_{c}=1.0,0.9,0.8,0.7,0.6$, and 0.5 . The stochastic simulations have been performed with $N_{p a r}=20,000$ parcels and the number of sections for discretizing the fluid velocity space is $N_{\text {sec }}=10$.

Fig. 14 shows the particle kinetic energy and the fluid-particle covariance with respect to the inverse of the Stokes number. As expected the standard Monte-Carlo algorithm predicts particle kinetic energy and fluid-particle covariance smaller than the DPS/DNS and the moment method. In contrast the CCV algorithm gives predictions in accordance with the DPS/DNS and with the moment method.

$$
\tau_{f p}^{F} / \tau_{f @ p}^{t}=0.43
$$

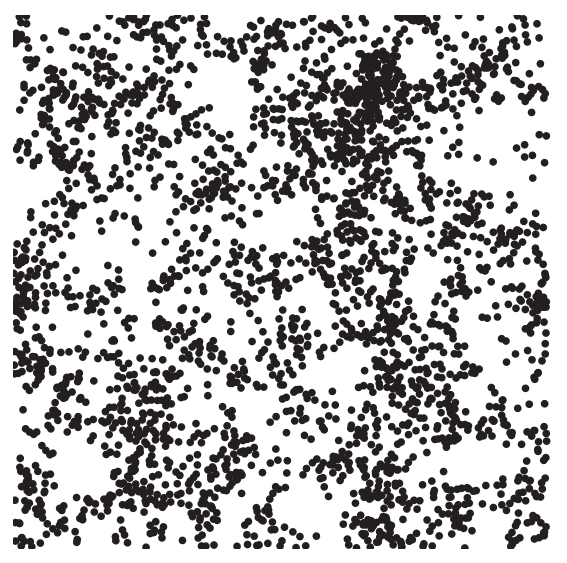

$$
\tau_{f p}^{F} / \tau_{f @ p}^{t}=1.70
$$

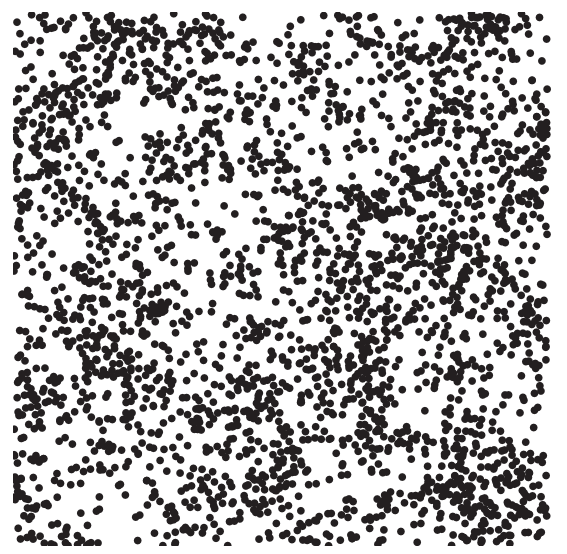

Fig. 13. Particle distribution in DPS/DNS. 

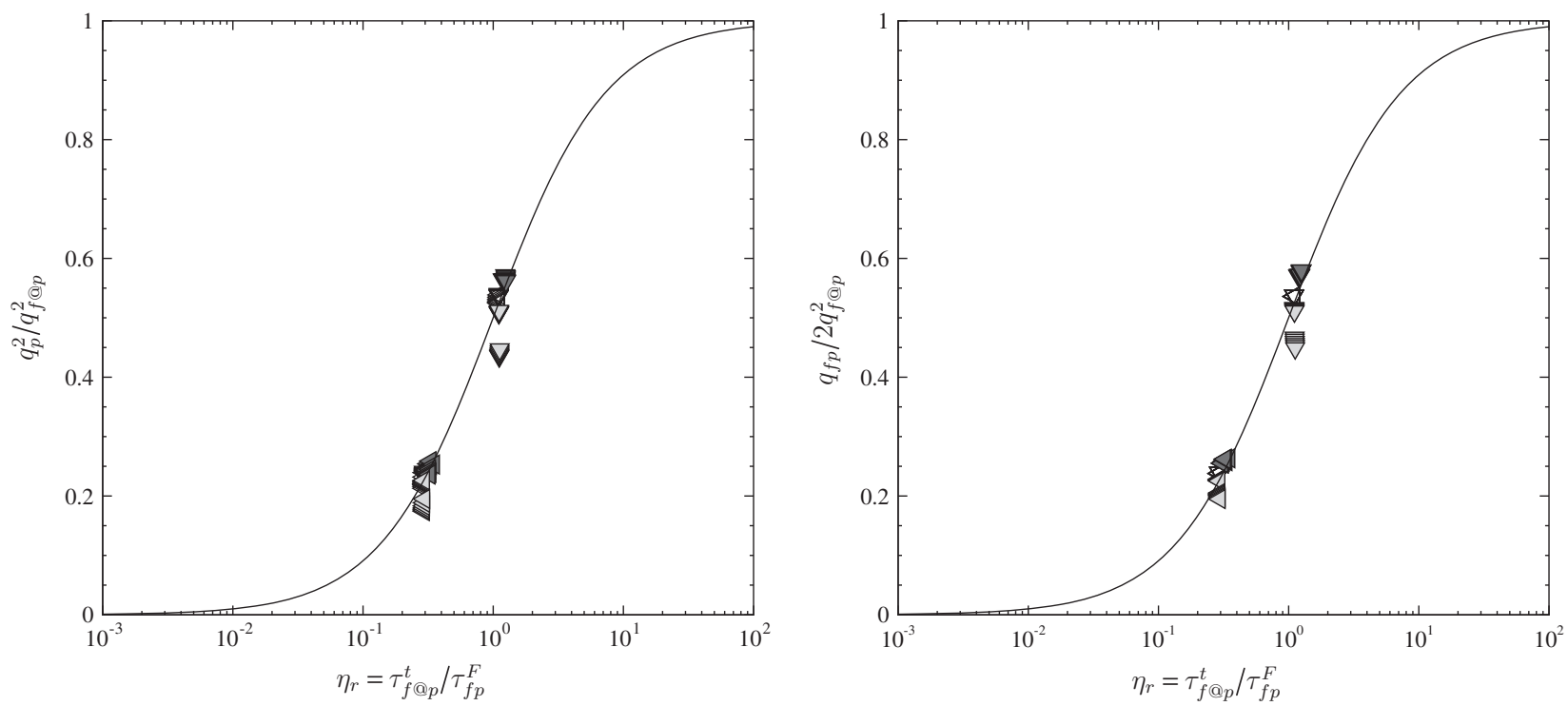

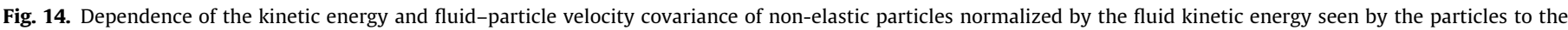

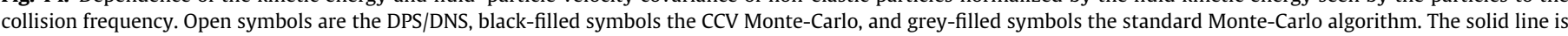
the prediction given by Eq. (38).

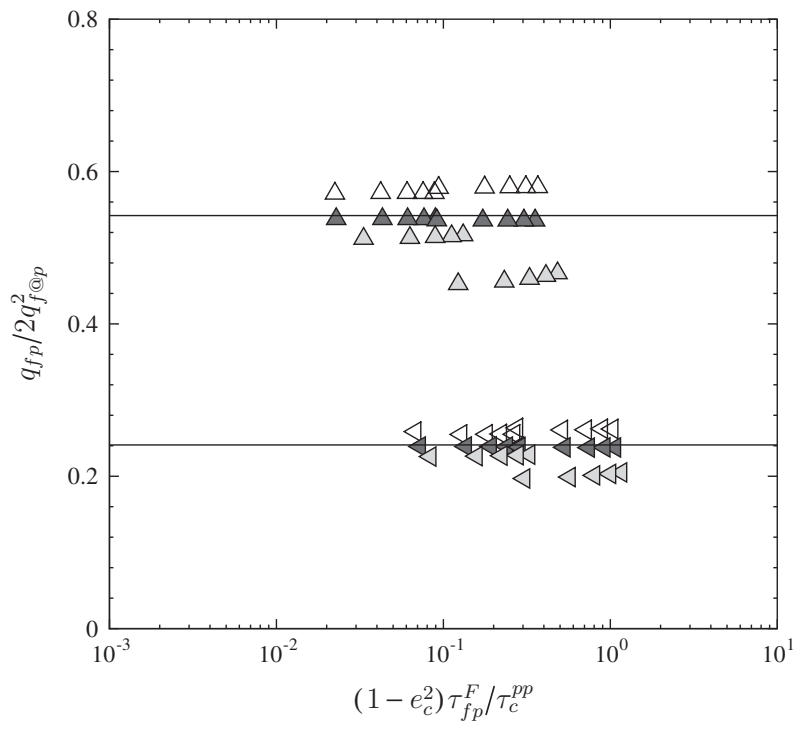

Fig. 15. Dependence of the fluid-particle velocity covariance of non-elastic particles normalized by the fluid kinetic energy seen by the particles to the collision frequency. Open symbols are the DPS/DNS, black-filled symbols the CCV Monte-Carlo, and grey-filled symbols the standard Monte-Carlo algorithm. The solid lines are the moment method predictions.

Fig. 15 shows the dependence of the fluid-particle velocity covariance to the collision frequency. As expected the fluid-particle covariance is conserved in DPS/DNS and in stochastic Monte-Carlo simulation using CCV. In contrast, the standard Monte-Carlo algorithm exhibits a destruction of $q_{f p}$. In the framework of the moment method, using correlated colliding velocity closure (16), for steady flow the particle kinetic energy is given by:

$q_{p}^{2}=q_{f @ p}^{2} \frac{\eta_{r}}{1+\eta_{r}}\left[1+\frac{\eta_{r}}{1+\eta_{r}} \frac{x}{6}\right] \times\left[1+\frac{x}{6}\right]^{-1}$

where $x=\left(1-e_{c}^{2}\right) \eta_{c}$ is a collision frequency. Fig. 16 shows that the particle kinetic energy predicted by the CCV Monte-Carlo

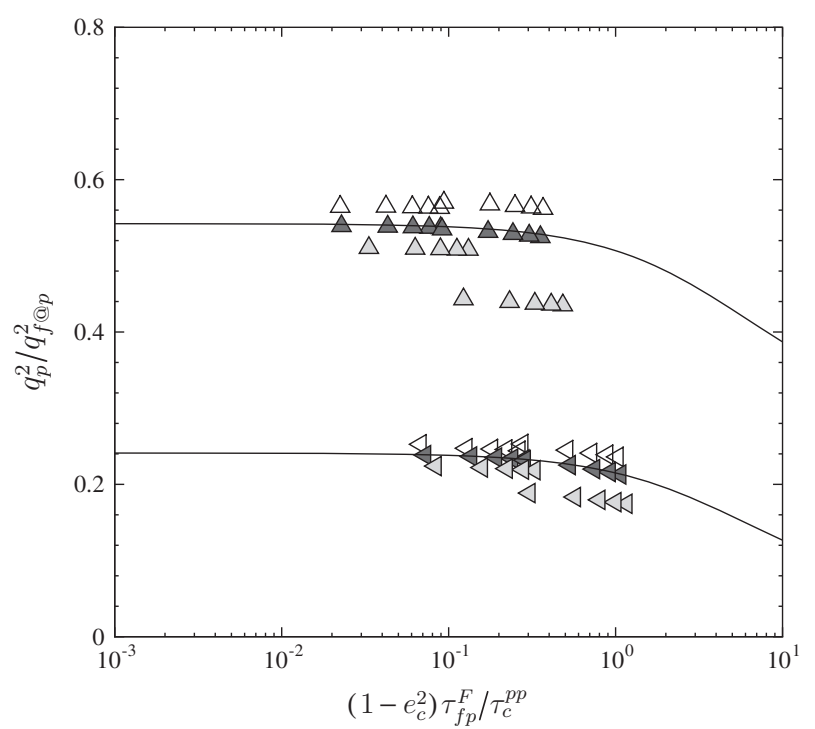

Fig. 16. Dependence of the kinetic energy of non-elastic particles normalized by the fluid kinetic energy seen by the particles to the collision frequency. Open symbols are the DPS/DNS, black-filled symbols the CCV Monte-Carlo, and grey-filled symbols the standard Monte-Carlo algorithm. The solid lines are the moment method predictions Eq. (42).

simulations are in accordance with the moment method. A good agreement is also found with DPS/DNS.

\section{Binary mixture of colliding particles}

Binary mixture of solid particles suspended in turbulent flows involves complex physical phenomena. Indeed, as in the monodisperse case, the interaction of solid particles with turbulent flow leads to transfers of kinetic energy from the gas towards the particles, but in a binary mixture the inter-particle collision between different species leads also to kinetic energy transfers between the solid phases (Pigeonneau, 1998; Fede and Simonin, 2003). Obviously the last transfers are controlled by the collision 
frequency between particle species and the Monte-Carlo algorithm has to reproduce this phenomenon. More, in the case of settling particles, the fluid-particle interactions lead to a mean particleparticle slip velocity between the solid species and then to the production of particle kinetic energy (Gourdel et al., 1998; Zaichik et al., 2009).

Binary mixture of elastic particles suspended in an homogeneous isotropic turbulent flows

Binary mixture of particles suspended in homogeneous isotropic turbulent flow has been numerically investigated by Fede and Simonin (2003) with DPS/LES simulations. Material properties and statistics of both the gas (air with a density $\rho_{f}=1.17 \mathrm{~kg} / \mathrm{m}^{3}$ and $\mu_{f}=1.72 \times 10^{-5} \mathrm{~kg} / \mathrm{m} / \mathrm{s}$ ) and the particles are gathered in Table 2 .

The Monte-Carlo simulations have been carried out with 20,000 parcels split in two groups of 10,000 parcels. Then the two solid species have the same number of parcels but different numerical weights. The fluid velocity space is discretized in 10 sections for the CCV Monte-Carlo algorithm.

In the framework of the kinetic theory of granular media, the mean collision time scales of a $p$-particle with any $q$-particle, $\tau_{k}^{p q}$, can be derived. Introducing the collision diameter, $d_{p q}=$ $\left(d_{p}+d_{q}\right) / 2$ this collision time scale writes

Table 2

Gas and particle material properties for mixtures of binary particles suspended in homogeneous isotropic turbulent flow.

\begin{tabular}{lll}
\hline & $p$-particles & $q$-particles \\
\hline Particle properties & & \\
Diameter, $d_{p}(\mu \mathrm{m})$ & 500 & 500 \\
Density, $\rho_{p}\left(\mathrm{~kg} / \mathrm{m}^{3}\right)$ & 75.0 & 300.0 \\
Particle restitution coefficient, $e_{c}$ & 1.0 & 1.0 \\
Volume fraction, $\alpha_{p}(-)$ & $1.56 \times 10^{-3}$ & Variable \\
Fluid turbulence seen, $q_{f @ p}^{2}\left(\mathrm{~m}^{2} / \mathrm{s}^{2}\right)$ & $85.4 \times 10^{-3}$ & \\
Fluid integral time scale seen, $\tau_{f @ p}^{t}(\mathrm{~s})$ & $39.4 \times 10^{-3}$ & \\
\hline
\end{tabular}

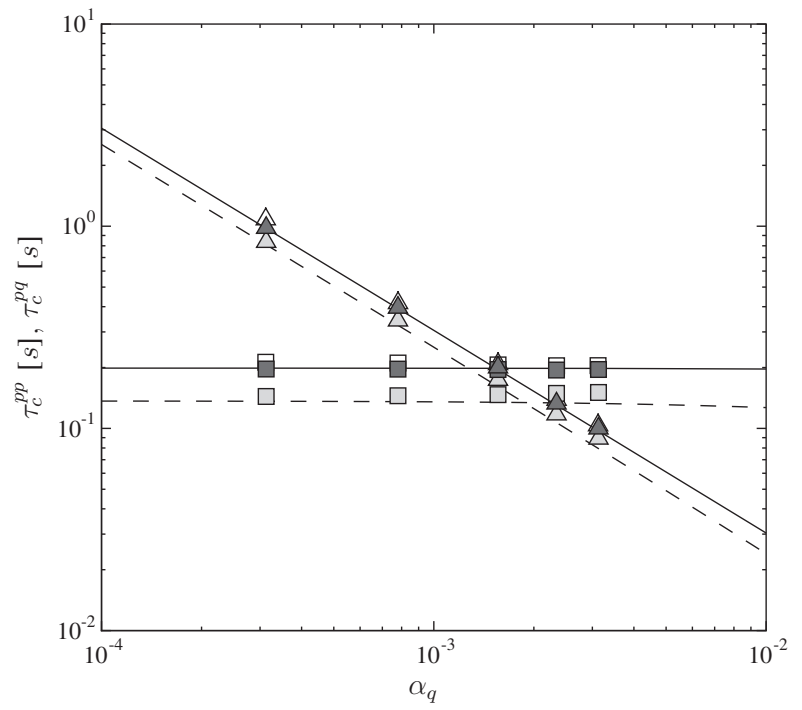

Fig. 17. Dependence of the mean collision time scale of a $p$-particle with all $p$ particles, $\tau_{c}^{p p}$, (squares) and with any $q$-particles, $\tau_{c}^{p q}$, (triangles up) measured in binary mixture of particles suspended in homogeneous isotropic turbulence. Open symbols are the DPS/LES performed by Fede and Simonin (2003), the grey-filled symbols the standard Monte-Carlo algorithm and the black-filled symbols the CCV Monte-Carlo algorithm. The dashed lines are the moment method based on the molecular chaos assumption Eq. (43) and the solid lines based on the CCV closure Eq. (44).

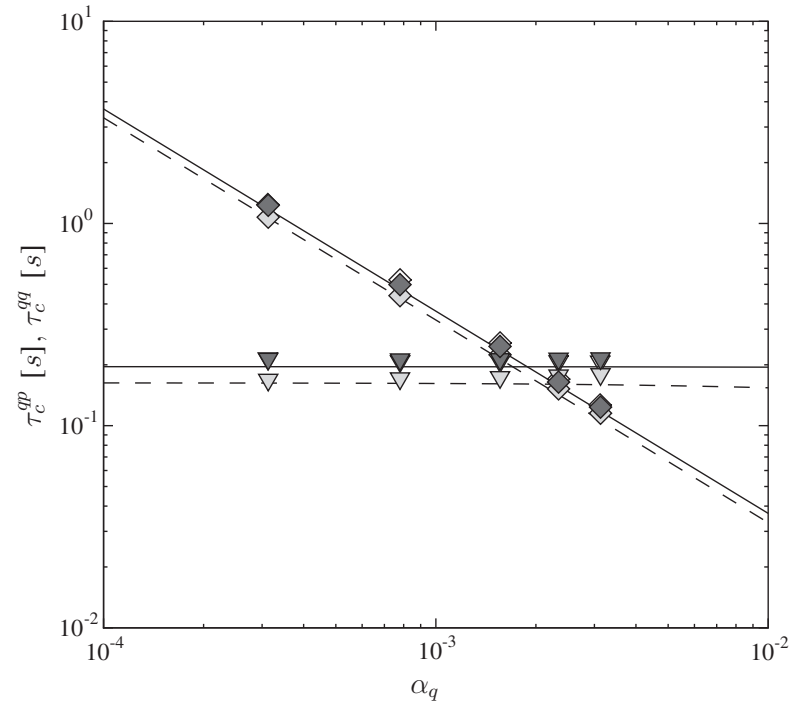

Fig. 18. Dependence of the mean collision time scale of a $q$-particle with all $q$-particles, $\tau_{c}^{q q}$, (diamonds) and with any $p$-particles, $\tau_{c}^{p q}$, (triangle down) measured in binary mixture of particles suspended in homogeneous isotropic turbulence. Open symbols are the DPS/LES performed by Fede and Simonin (2003), the greyfilled symbols the standard Monte-Carlo algorithm and the black-filled symbols the CCV Monte-Carlo algorithm. The dashed lines are the moment method based on the molecular chaos assumption Eq. (43) and the solid lines based on the CCV closure Eq. (44)

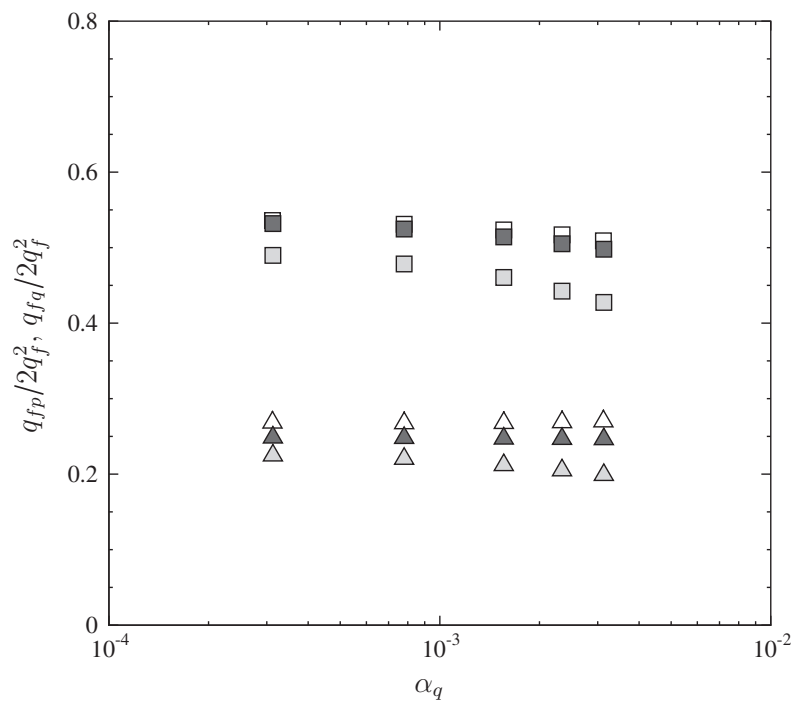

Fig. 19. Dependence of the fluid-particle velocity covariance of $p$-particles (squares) and $q$-particles (triangles) suspended in homogeneous isotropic turbulence. Open symbols are the DPS/LES performed by Fede and Simonin (2003), the grey-filled symbols the standard Monte-Carlo algorithm and the black-filled symbols the CCV Monte-Carlo algorithm. The dashed lines are the moment method based on the molecular chaos assumption and the solid lines based on the CCV closure.

$\frac{1}{\tau_{k}^{p q}}=\pi d_{p q}^{2} n_{q} \sqrt{\frac{16}{\pi} \frac{1}{3}\left(q_{p}^{2}+q_{q}^{2}\right)}$.

Pigeonneau (1998) derived an expression taking into account the correlations induced by the particle-turbulence interactions:

$\frac{1}{\tau_{c}^{p q}}=\pi d_{p q}^{2} n_{q} \sqrt{\frac{16}{\pi} \frac{1}{3}\left(q_{p}^{2}+q_{q}^{2}-2 \sqrt{\xi_{f p}^{2} \xi_{f q}^{2} q_{p}^{2} q_{q}^{2}}\right]}$

where $\xi_{f p}^{2}$, respectively $\xi_{f q}^{2}$, is the correlation coefficient of the $p$-particle, respectively the $q$-particles. Figs. 17 and 18 show 
inter-particle collision time scales with respect to the solid volume fraction of $q$-particles. As expected, on one hand, for increasing $\alpha_{q}$ the collision time scale $\tau_{c}^{p q}$ is decreasing because of increasing the collision frequency. On another hand, the collision time scale $\tau_{c}^{p p}$ is nearly constant. Figs. 17 and 18 show that the standard Monte-Carlo algorithm overestimates the collision frequency and consequently underestimates the collision time scale. In contrast the predictions of CCV Monte-Carlo algorithm are in accordance with the DPS/LES results. Also Figs. 17 and 18 depict that the predictions of the moment method are improved with CCV closure).

Fig. 19 shows the fluid-particle velocity covariance for the two solid species with respect to the solid volume fraction of the $q$-particles. The fluid-particle covariance of $p$-particles is found decreasing for increasing the collision frequency. For the standard Monte-Carlo algorithm this trend was expected because of the covariance destruction induced by the molecular chaos assumption. However, a small decrease of $q_{f p}$ is also observed in DPS/LES results and for the predictions using CCV Monte-Carlo algorithm. The reason of the modification of the fluid-particle covariance is a physical phenomenon that is a transfer of covariance by collision taking place only in binary mixture of particles.

Fig. 20 shows the particle kinetic energy of the two solid phases with respect to the solid volume fraction of $q$-particles. The DPS/LES results exhibit a small decrease of $p$-particle kinetic energy due to the observed decrease of the fluid-particle velocity

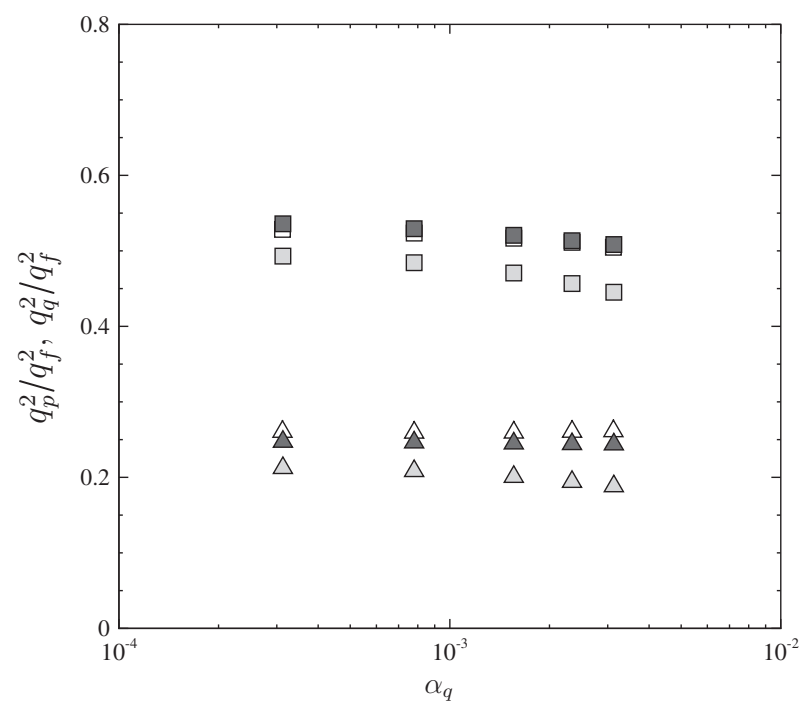

Fig. 20. Dependence of the kinetic energy of $p$-particles (squares) and $q$-particles (triangles) suspended in homogeneous isotropic turbulence. Open symbols are the DPS/LES performed by Fede and Simonin (2003), the grey-filled symbols the standard Monte-Carlo algorithm and the black-filled symbols the CCV Monte-Carlo algorithm. The dashed lines are the moment method based on the molecular chaos assumption and the solid lines based on the CCV closure.

\section{Table 3}

Gas and particle material properties for mixtures of binary particles falling in homogeneous isotropic turbulent flow (as in DPS/LES the gravity is set to $-49.05 \mathrm{~m} / \mathrm{s}^{2}$ ).

\begin{tabular}{lll}
\hline & $p$-particles & $q$-particles \\
\hline Particle properties & & \\
Diameter, $d_{p}(\mu \mathrm{m})$ & 650 & 650 \\
Density, $\rho_{p}\left(\mathrm{~kg} / \mathrm{m}^{3}\right)$ & 117.5 & 235.0 \\
Particle restitution coefficient, $e_{c}$ & 1.0 & 1.0 \\
Volume fraction, $\alpha_{p}(-)$ & $13.1 \times 10^{-3}$ & Variable \\
Fluid turbulence seen, $q_{f @ p}^{2}\left(\mathrm{~m}^{2} / \mathrm{s}^{2}\right)$ & 0.131 & 0.131 \\
Fluid integral time scale seen, $\tau_{f @ p}^{t}(\mathrm{~s})$ & $23.0 \times 10^{-3}$ & $23.0 \times 10^{-3}$ \\
\hline
\end{tabular}

covariance shown by Fig. 19. As expected, Fig. 20 shows that the standard Monte-Carlo algorithm leads to a strong underestimation of the particle kinetic energy. In the binary mixture, the molecular chaos assumption has several consequences. The molecular chaos assumption overestimates the collision frequency, see Fig. 17, and also the transfer rate of particle kinetic energy from one particle specie to the other one.

Binary mixture of elastic particles falling in homogeneous isotropic turbulent flows

The settling of a binary mixture of particles in homogeneous isotropic turbulence has been investigated by Gourdel et al.

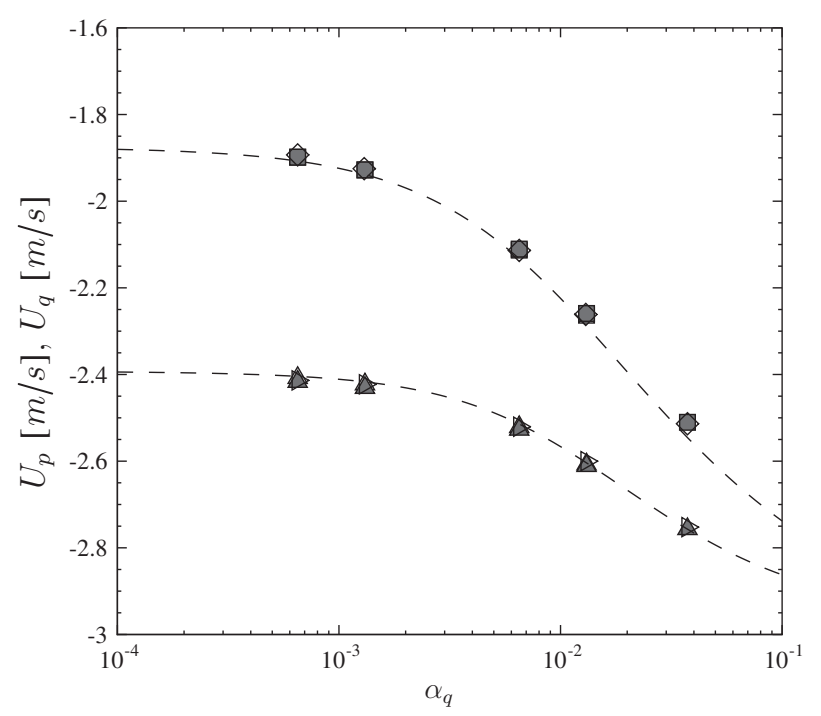

Fig. 21. Dependence of the mean vertical particle velocities of a binary mixture of particles settling in homogeneous isotropic turbulence. The open symbols are the DPS/LES performed by Gourdel et al. (1998), the grey-filled symbols the standard Monte-Carlo algorithm and the black-filled symbols the CCV Monte-Carlo algorithm. The solid lines are the predictions of the moment method with the molecular chaos assumption.

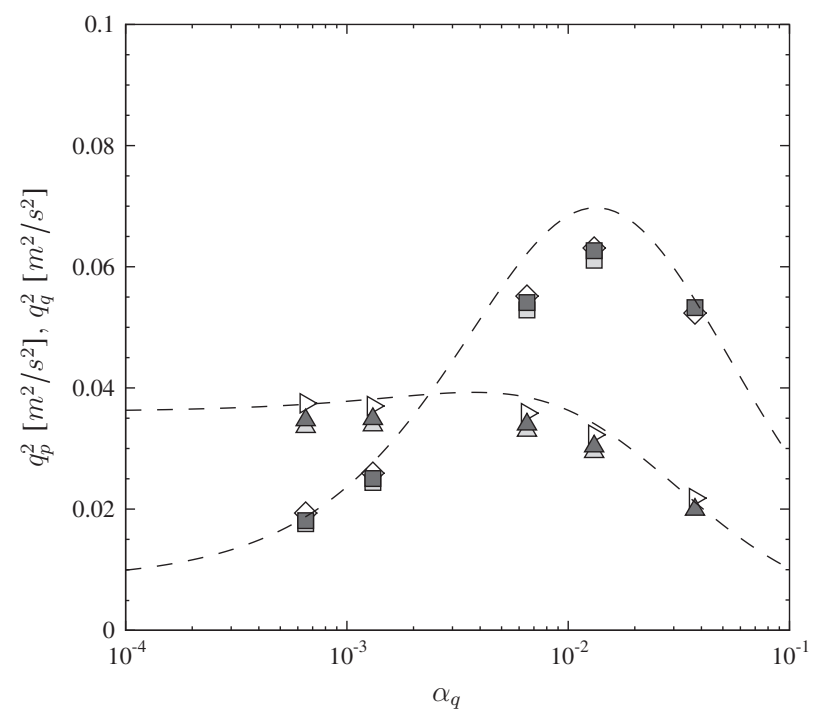

Fig. 22. Dependence of the mean particle kinetic energy of particle of a binary mixture of particles settling in homogeneous isotropic turbulence. The open symbols are the DPS/LES performed by Gourdel et al. (1998), the grey-filled symbols the standard Monte-Carlo algorithm and the black-filled symbols the CCV MonteCarlo algorithm. The solid lines are the predictions of the moment method with the molecular chaos assumption. 
Table 4

Gas and droplet material properties for numerical simulations of coalescing droplets.

\begin{tabular}{|c|c|c|c|}
\hline \multicolumn{4}{|l|}{ Fluid properties } \\
\hline Reynolds number, $R e$ & & & 61.2 \\
\hline Reynolds number, $R e_{\lambda}$ & & & 32.9 \\
\hline Turbulence agitation, $q_{f}^{2}\left(\mathrm{~m}^{2} / \mathrm{s}^{2}\right)$ & & & $67 \times 10^{-3}$ \\
\hline Turbulent time scale, $\tau_{f}^{t}(\mathrm{~s})$ & & & $14.3 \times 10^{-3}$ \\
\hline Symbols & $\square$ & $\triangle$ & $\diamond$ \\
\hline \multicolumn{4}{|l|}{ Droplet properties at $t=0$} \\
\hline Diameter, $d_{p}(\mu \mathrm{m})$ & 150 & & \\
\hline Volume fraction, $\alpha_{p}(-)$ & $8.3 \times 10^{-5}$ & & \\
\hline Stokes number, $\tau_{p} / \tau_{f}^{t}$ & 0.11 & 1.01 & 3.00 \\
\hline
\end{tabular}

(1998) by performing DPS/LES. In such case, the turbulence is statistically steady by using a forcing. Material properties and statistics of both the gas (air with a density $\rho_{f}=1.17 \mathrm{~kg} / \mathrm{m}^{3}$ and $\mu_{f}=1.72 \times 10^{-5} \mathrm{~kg} / \mathrm{m} / \mathrm{s}$ ) and the particles are gathered in Table 3 .

When particles are falling through a turbulent the fluid integral time scale seen by the particles is modified by the mean fluid-particle relative velocity (Yudine, 1959). Csanady (1963) proposed the

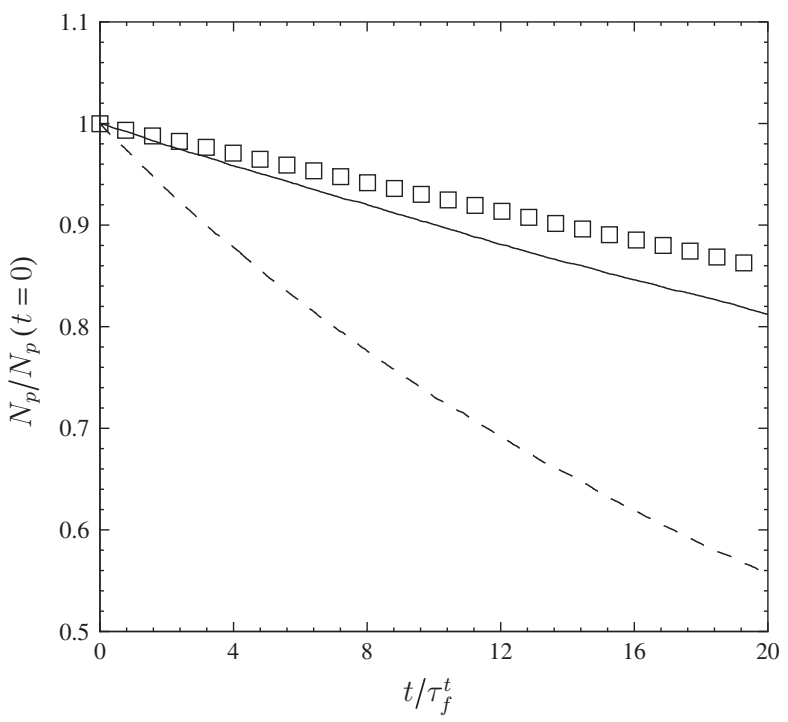

following model for the integral time scale of the turbulence seen by falling particles:

$$
\tau_{f @ p, \|}^{t}=\frac{\tau_{f}^{t}}{\sqrt{1+\left(\frac{L_{f} V_{r}}{\tau_{f}^{t}}\right)^{2}}} \text { and } \tau_{f @ p, \perp}^{t}=\frac{\tau_{f}^{t}}{\sqrt{1+4\left(\frac{L_{f} V_{r}}{\tau_{f}^{t}}\right)^{2}}}
$$

where $L_{f}$ is the longitudinal integral length scale of the turbulence, $V_{r}$ is the mean gas-particle relative velocity. The time scale $\tau_{f @ p, \|}^{t}$, respectively $\tau_{f @ p, \perp}^{t}$, is the Lagrangian integral time scale of the fluid velocity seen by the particles in parallel, resp. perpendicular, direction of the mean gas-particle relative velocity. Following Simonin et al. (1993) in stochastic simulations the Langevin equation (B.1) is modified accordingly to (45). As previously, the Monte-Carlo simulations have been performed with 20,000 parcels split in two equal groups of 10,000 parcels and the fluid velocity space is discretized in 10 sections for the CCV Monte-Carlo algorithm.

The mean vertical particle velocities are shown by Fig. 21. As expected the $q$-particles, namely the heaviest, are settling with a larger velocity than the $p$-particles. In a binary mixture, the inter-specie collisions lead to momentum transfers between solid

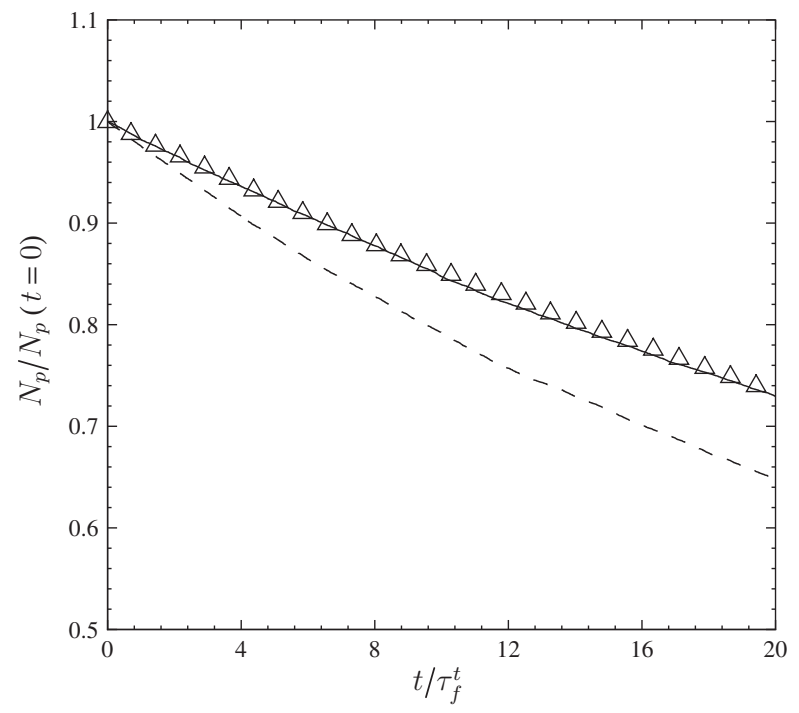

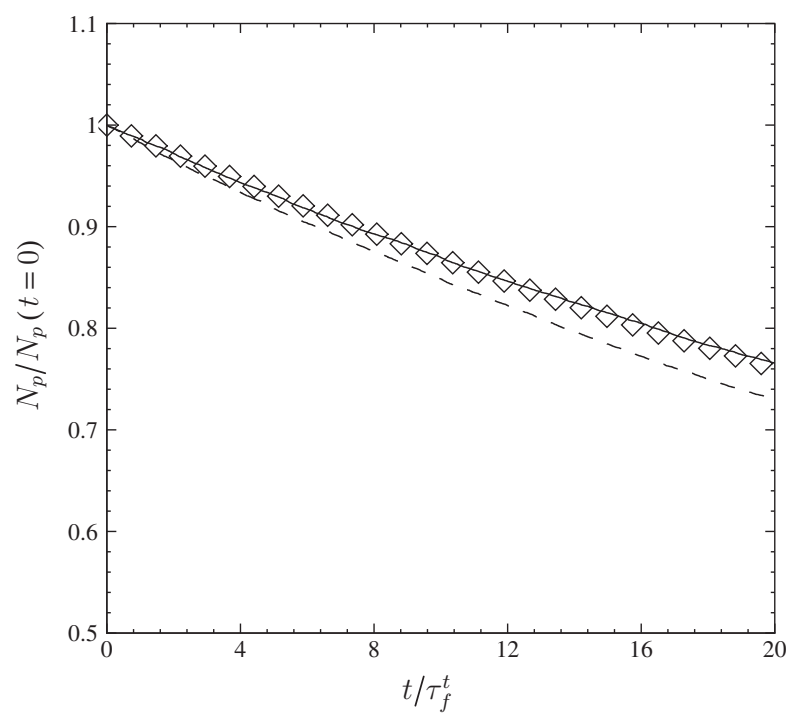

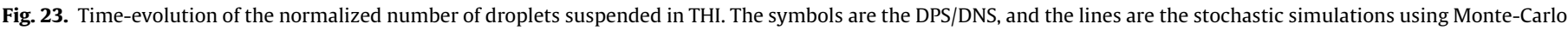
algorithm (solid lines: CCV algorithm and dashed lines: standard algorithm). 

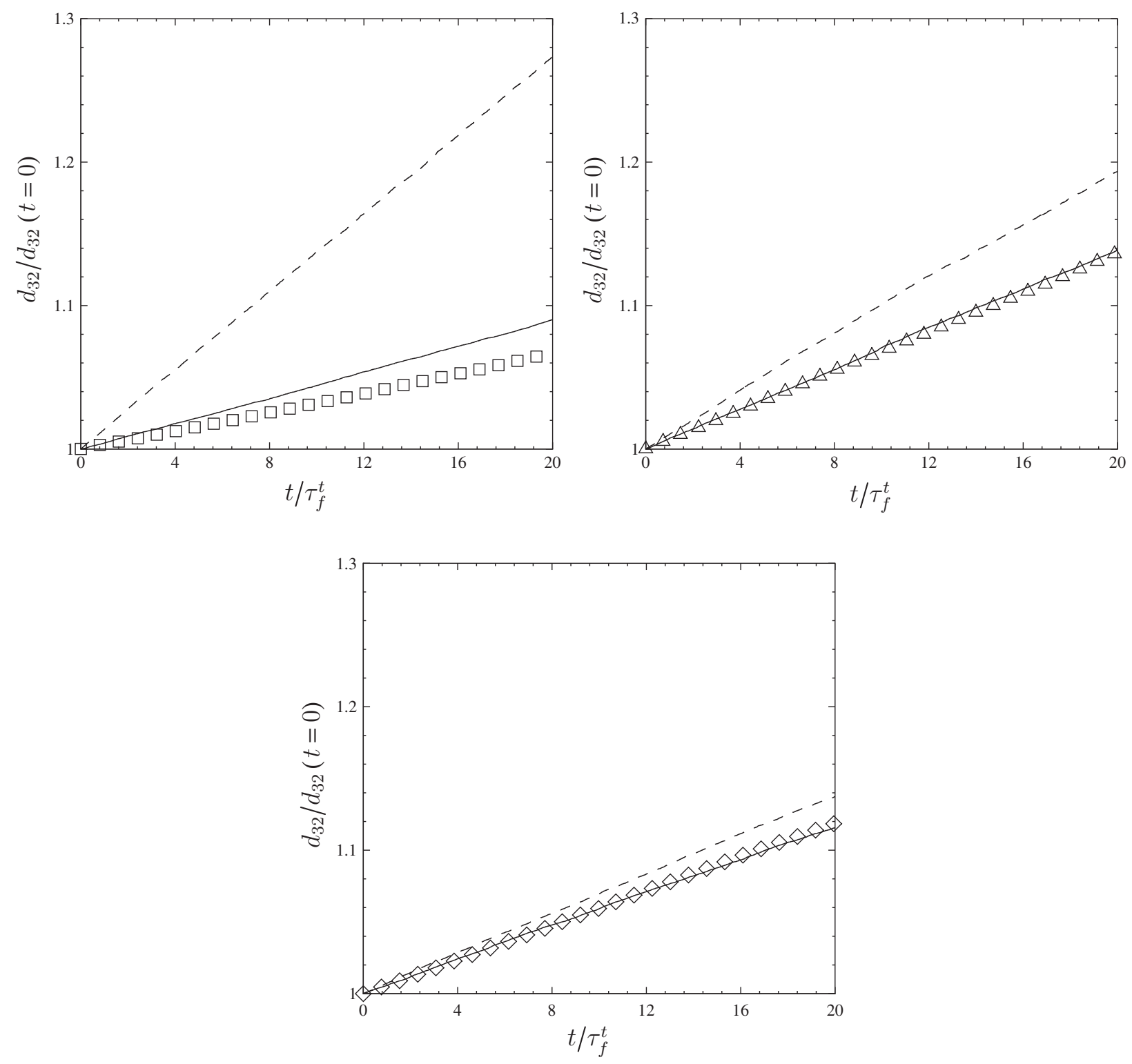

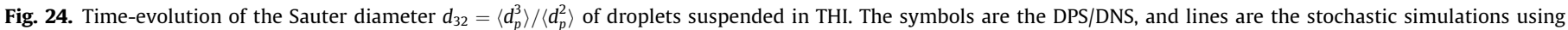
Monte-Carlo algorithm (solid lines: CCV algorithm and dashed lines: standard algorithm).

phases and increasing the solid volume fraction of $q$-particle leads to increase the momentum transfer. As a consequence for large value of $\alpha_{q}$ the mean particle velocities are closer and closer. Fig. 21 shows that the Monte-Carlo simulations are both, using the standard algorithm or the CCV algorithm, in accordance with the moment approach (Gourdel et al., 1998; Zaichik et al., 2009) based on molecular chaos assumption meaning that in such a case the correlations induced by the turbulence are not important.

The particle kinetic energies with respect to the solid volume fraction of $q$-particles are shown by Fig. 22. It can be noticed that the stochastic simulations agree very well with the DPS/LES meaning that the production of kinetic energy by the mean particle-particle relative velocity is well taken into account in stochastic simulations. The small differences with the moment method prediction are probably due to the anisotropy of the fluctuating motion of the particle velocity. Indeed, the moment method used for Figs. 21 and 22 is based on a $q_{p}^{2}-q_{f p}$ model meaning that, for each solid phase, only one equation for the particle kinetic energy and one equation for the fluid-particle covariance are solved. In the present case, the anisotropy of the particle velocity may require a second order moment approach solving the equations of the particle kinetic stress tensor.

\section{Coalescing droplets}

In this section we use the CCV Monte-Carlo algorithm for the prediction of the coalescence of droplets transported by a homogeneous isotropic turbulent flow. As for the collision of solid particle, the numerical results from Monte-Carlo simulations are compared with results obtained by DPS/DNS for coalescing droplets (Wunsch et al., 2010b,a). As previously explained, in the present study we consider only the permanent coalescence regime. Then mass of the new droplet and its momentum are given by Eq. (9) and (10).

As for the solid colliding particles, the computational domain is a cubic box of length $L_{b}=0.128 \mathrm{~m}$ with full periodic boundary conditions. The fluid is air with kinematic viscosity $v_{f}=1.47 \times$ $10^{-5} \mathrm{~m}^{2} \mathrm{~s}^{-1}$ and density $\rho_{f}=1.17 \mathrm{~kg} \mathrm{~m}^{-3}$. All relevant physical properties before starting the coalescence are gathered in Table 4. 
The numerical simulations are performed as following. First we perform a simulation without the coalescence. During this phase the interaction of the droplets with the turbulence leads the droplet to be agitated respecting to the local equilibrium (TchenHinze theory). Then the coalescence is activated. The statistics before the coalescence are gathered in Table 4.

As expected and shown by Figs. 23 and 24 the coalescence leads to the decrease of the number of droplets and increase the droplet diameter. Fig. 23 shows the time-evolution of the droplet number for three Stokes numbers. For the largest Stokes number $(S t=3.00)$ the molecular chaos assumption and the CCV Monte-Carlo algorithm predict nearly the same behaviour. In such a case, the correlations induced by the turbulence on the two neighbouring particles are not important. In contrast, for small values of St the molecular chaos (dashed line) overestimates the rate of vanishing droplets by the overestimation of the collision frequency. For $S t=1.01$ the predictions given by the CCV algorithm are in good accordance with the results by a deterministic approach. For small Stokes number $S t=0.11$, compare to the molecular chaos assumption the CCV algorithm strongly improves the results. However the coalescence rate is a little bit larger than the one measured in DNS. This difference may be explained from the high level of preferential concentration that occurs for such a Stokes number (Shaw et al., 1998).

\section{Conclusions}

A new Monte-Carlo algorithm has been proposed for the prediction of particle- or droplet-laden turbulent flows in framework of the RANS approach. The algorithm allows to solve rigorously the kinetic equation from a joint fluid-particle pdf approach. In comparison with other algorithms found in the literature the new approach allows a strict conservation of the fluid-particle covariance without any assumption. The new algorithm has been assessed on several configurations: elastic monodisperse particles, non-elastic monodisperse particles, binary mixture of elastic particles and binary mixture of elastic settling particles in turbulent flow and finally coalescing droplets. The predictions given by the Monte-Carlo algorithm are in very good accordance with deterministic results from DPS/DNS or DPS/LES and predictions with moment method. However, it is known that the theoretical closure proposed by Laviéville et al. (1995) leads to an underestimation of the correlation induced by the interaction of particles with the turbulence. This effect is partially compensated because the effect of spatial concentration is not taken into account as usual in all Monte-Carlo algorithms.

For practical application the computational cost is essentially the discretization of the fluid velocity realization space that requires a large number of parcels compared to the standard algorithms. However, the main goal of the paper is to demonstrate the feasibility of the method and its assessment by comparison with academic cases from literature. The choice of a uniform mesh for the discretization of the fluid velocity space can be optimized in order to reduced the computational cost.

Even if the validations have been all performed for particles suspended in homogeneous isotropic turbulent flow the method developed in the paper is not limited by that. Indeed, the nature of the flow carrying the particles or the droplets is not a parameter of the algorithm and the particle fluctuating motion is not supposed to be in local equilibrium with the fluid turbulence. Extension to more complex turbulent fluid flow may be directly carried out by changing the stochastic model representing the fluid turbulence along the particle paths such as the ones proposed by Sawford (1991) or Pope (2002) for low Reynolds number flows or homogeneous shear flows, respectively.
The Monte-Carlo algorithm described in the paper is developed in the framework of the coupling between fluid RANS turbulence and particle stochastic Lagrangian modelling approaches to account for the particle velocity correlation induced by the interaction with the whole turbulence energy spectrum. Nevertheless, as pointed by Fede and Simonin (2006), in the DPS/LES prediction, the unresolved fluid turbulence may induce a correlation between velocities of colliding particles with relaxation time equal to or smaller than the subgrid turbulent time scale. Therefore, the proposed Monte-Carlo algorithm should be extended to such DPS/LES approach by using a Lagrangian stochastic process to represent the subgrid fluid turbulence along the particle paths (Fede et al., 2006).

\section{Appendx A. Deterministic particle Lagrangian simulation coupled with Direct Numerical Simulation of the gas turbulence}

The fluid flow is governed by incompressible Navier-Stokes equations discretized using a finite-volume method on staggered mesh with a second-order centred scheme (Magnaudet et al., 1995). The time-integration is performed with a second order Runge \& Kutta scheme. Turbulent statistically steady flow is obtained with a stochastic spectral forcing proposed by Eswaran and Pope (1988). The dispersed phase is composed of $N_{p}$ solid, spherical, identical particles or droplets. As already explained the forces acting on the particles are reduced to only the drag force. Then the single particle motion governing equation writes

$$
\begin{aligned}
\frac{d \mathbf{x}_{p}}{d t} & =\mathbf{u}_{p} \\
\frac{d \mathbf{u}_{p}}{d t} & =-\frac{\mathbf{u}_{p}-\mathbf{u}_{f @ p}}{\tau_{p}}+\mathbf{g}
\end{aligned}
$$

where $\mathbf{x}_{p}$ and $\mathbf{u}_{p}$ are the particle position and velocity and $\mathbf{g}$ the gravity that could be neglected in some cases. The particle response time, $\tau_{p}$, is defined as

$\tau_{p}=\frac{\rho_{p}}{\rho_{f}} \frac{4}{3} \frac{d_{p}}{C_{d}} \frac{1}{\left|\mathbf{u}_{p}-\mathbf{u}_{f @ p}\right|}$.

As the two-way coupling is not considered, the undisturbed fluid velocity $\mathbf{u}_{f @ p}(t)=\mathbf{u}_{f}\left(\mathbf{x}_{p}(t), t\right)$ is given directly by the DNS and evaluated at the particle position by a cubic spline interpolation scheme. The drag coefficient, $C_{d}$, is given by Schiller and Nauman (1935), $C_{d}=24 / \operatorname{Re}_{p}\left[1+0.15 R e_{p}^{0.687}\right]$ with the particle Reynolds number given by, $R e_{p}=d_{p}\left|\mathbf{u}_{p}-\mathbf{u}_{f @ p}\right| / v_{f}$. For the analysis, the mean particle response time $\tau_{f p}^{F}$ is introduced. It is defined as $1 / \tau_{f p}^{F}=\left\langle 1 / \tau_{p}\right\rangle_{p}$ with the particle average operator $\langle\cdot\rangle_{p}$. The numerical method used for the calculation of particle positions Eq. (A.1) and velocities Eq. (A.2) is a second-order Runge \& Kutta scheme with the same time step than the DNS.

For the study on monodisperse particles and for the droplet-laden flows the fluid flow was predicted by DNS. For the case of the binary mixture of particles, the fluid flow was predicted by using LES where the subgrid viscosity was modelled with the dynamic mixed model proposed by Germano et al. (1991). In case of DPS/LES no effect of the subgrid velocity is accounted for in the particle trajectory equation according to Fede and Simonin (2006) where it is shown that for particles having a sufficient inertia this effect is negligible.

Several algorithms for the treatment of the collisions in dispersed phase are found in literature (Hopkins and Louge, 1991; Sundaram and Collins, 1997; Sakiz and Simonin, 1998; Sigurgeirsson et al., 2001). The simplest, but also an extremely inefficient way to detect all neighbouring particles is to compute all inter-particle distances. With such a method the numerical cost 
of checking for collision of the order of $O\left(N_{p}^{2}\right)$. The computation cost can be reduced by using a detection grid (Hopkins and Louge, 1991). Such an algorithm may reduce the computation cost to $O\left(124 N_{p}\right)$. For polydisperse particle mixtures and coalescence of droplets Wunsch et al. (2008) proposed and implemented a collision detection algorithm which was used in this work. More details can be found in Wunsch et al. (2010a). When colliding particles are detected, the post-collisional velocities are computed in the frame of the hard sphere collision model following Eqs. (7) and (8). For coalescing droplets, the mass and velocity of the resulting droplet are computed according to Eqs. (9) and (10) (corresponding to mass and momentum conservation).

\section{Appendix B. Stochastic particle and gas turbulence Lagrangian simulation}

The parcels are time-advanced by solving the same set of Eqs. (A.1) and (A.2) than in DPS/DNS. As previously mentioned the prediction of the fluid turbulent velocity along the trajectory of inertial particle is a complex challenge (Pialat et al., 2007). In case a homogeneous isotropic turbulent flows the Langevin equation proposed by Pope (1994) is efficient. Simonin et al. (1993) proposed the following extension for the fluid velocity seen by inertial particles:

$\frac{d u_{f @ p, i}}{d t}=-\frac{u_{f @ p, i}}{\tau_{f @ p}^{t}}+\sqrt{\frac{4}{3} \frac{q_{f @ p}^{2}}{\tau_{f @ p}^{t}}} \delta W_{i}$

where $\tau_{f @ p}^{t}$ is the turbulent Lagrangian integral time scale seen by the particles and $\delta W_{i}$ a Wiener process.

High-order time integration of stochastic equation is quite complex (Minier and Peirano, 2001) but seems to be unnecessary in simple flows, such as homogeneous isotropic turbulence, if the computational time step is small enough with respect to the turbulent Lagrangian integral time scale (in all stochastic simulations the time step is such as $\left.\Delta t / \tau_{f @ p}^{t}<100\right)$. Then a first order Euler scheme is used for the time integration. It leads to the following numerical scheme for predicting the fluid velocity of each parcel

$u_{f @, i}^{n+1}=u_{f @, i}^{n}\left[1-\frac{\Delta t}{\tau_{f @ p}^{t}}\right]+\sqrt{\frac{4}{3} \frac{q_{f @ p}^{2}}{\tau_{f @ p}^{t}} \Delta t}$

where $\zeta$ is a random variable following a normalized Gaussian distribution. As Eq. (B.1) is a Stochastic Differential Equation the integration scheme is a Euler-Maruyama scheme consistent with an Ito integration (Kloeden and Platen, 1992).

In the DPS/RANS numerical simulations, the fluid turbulent time scale and kinetic energy used in the Lagrangian stochastic process (B.2) are assumed to be independent of the Stokes number value $\left(\tau_{f @ p}^{t}=\tau_{f}^{t}\right.$ and $\left.q_{f @ p}^{2}=q_{f}^{2}\right)$.

\section{References}

Abrahamson, J., 1975. Collision rates of small particles in a vigorously turbulent fluid. Chem. Eng. Sci. 30, 1371-1379.

Ashgriz, N., Poo, J.Y., 1990. Coalescence and separation in binary collisions of liquid drops. J. Fluid Mech. 221, 183-204.

Babovsky, H., 1986. On a simulation scheme for the Boltzmann equation. Math. Methods Appl. Sci. 8, 223-233.

Balachandar, S., Eaton, J.K., 2010. Turbulent dispersed multiphase flow. Annu. Rev. Fluid Mech. 42 (1) 111-133.

Berlemont, A., Simonin, O., Sommerfeld, M., 1995. Validation of inter-particle collision models based on large eddy simulation. Gas-Solid Flows, vol. 228. ASME FED, pp. 359-369.

Berlemont, A., Achim, P., Chang, Z., 2001. Lagrangian approaches for particle collisions: the colliding particle velocity correlation in the multiple particles tracking method and in the stochastic approach. Phys. Fluids 13, 2946-2956.
Bird, G.A., 1969. Direct numerical and the Boltzmann equation. Phys. Fluids 11, 2676-2681.

Buyevich, Y.A., 1971. Statistical hydromechanics of disperse systems part 1. Physical background and general equations. J. Fluid Mech. 49, 489-507.

Buyevich, Y.A., 1972. Statistical hydromechanics of disperse systems. Part 2. Solution of the kinetic equation for suspended particles. J. Fluid Mech. 52, $345-355$.

Chapman, S., Cowling, T., 1970. The Mathematical Theory of Non-Uniform Gases Cambridge University Press, Cambridge.

Crowe, C., Sommerfeld, M., Tsuji, Y., 1998. Multiphase Flows with Droplets and Particles. CRC Press.

Csanady, G., 1963. Turbulent diffusion of heavy particles in the atmosphere. J. Atmos. Sci. 20, 201-208.

Derevich, I., Zaichik, L., 1988. Precipitation of particles from a turbulent flow Izvetiyia Akad. Nauk SSSR Mekh. Zhidkosti i Gaza 5, 96-104.

Deutsch, E., Simonin, O., 1991. Large eddy simulation applied to the motion of particles in stationary homogeneous fluid turbulence. Turbulence Modification in Multiphase Flows, vol. 110. ASME FED, pp. 35-42.

Eswaran, V., Pope, S., 1988. An examination of forcing in direct numerical simulations of turbulence. Comput. Fluids 16, 257-278.

Fede, P., Simonin, O., 2003. Modeling of kinetic energy transfer in a non-settling binary mixture of particles suspended in a turbulent homogeneous flows. In: 6th Int. Symp. on Gas-Particle Flows. ASME FEDSM2003-45735, pp. 483-490.

Fede, P., Simonin, O., 2006. Numerical study of the subgrid fluid turbulence effects on the statistics of heavy colliding particles. Phys. Fluids 18 (045103), 1-17.

Fede, P., Simonin, O., 2010. Effect of particle-particle collisions on the spatia distribution of inertial particles suspended in homogeneous isotropic turbulent flows. Turbulence and Interactions. Notes on Numerical Fluid Mechanics and Multidisciplinary Design, vol. 110. Springer, Berlin Heidelberg, pp. 119-125.

Fede, P., Simonin, O., Villedieu, P., Squires, K., 2006. Stochastic modeling of the turbulent subgrid fluid velocity along inertial particle trajectories. In: Proceedings of the Summer Program. Center for Turbulence Research.

Fessler, J., Kulick, J., Eaton, J., 1994. Preferential concentration of heavy particles in a turbulent channel flow. Phys. Fluids 6, 3742-3749.

Février, P., Simonin, O., Squires, K.D., 2005. Partitioning of particle velocities in gassolid turbulent flows into a continuous field and a spatially uncorrelated random distribution: theoretical formalism and numerical study. J. Fluid Mech. 533, 1-46.

Fox, R.O., 2012. Large eddy simulation tools for multiphase flows. Annu. Rev. Fluid Mech. 44 (1), 47-76.

Gatignol, R., 1983. The Faxen formulae for a rigid particle in an unsteady non uniform stokes flow. J. Mec. Th. Appl. 9, 143-160.

Germano, M., Piomelli, U., Moin, P., Cabot, W., 1991. A dynamic subgrid-scale eddy viscosity model. Phys. Fluids 3 (7), 1760-1765.

Gourdel, C., Simonin, O., Brunier, E., 1998. Modelling and simulation of gas-solid turbulent flows with a binary mixture of particles. In: Third Internationa Conference on Multiphase Flow. ICFM'98, Lyon, France.

Haworth, D., Pope, S., 1986. A generalized Langevin model for turbulent flows. Phys. Fluids 29, 387-404

Hopkins, M., Louge, M., 1991. Inelastic microstructure in rapid granular flows of smooth disks. Phys. Fluids 3, 47-57.

Ivanov, M., Rogasinsky, S., 1988. Analysis of numerical technics of the direct simulation Monte Carlo method in the rarefied gas dynamics. Sov. J. Num. Anal Math. Model. 3, 453-465.

Jenkins, J.T., Richman, M.W., 1985. Grad's 13-moments system for dense gas of inelastic spheres. Arch. Ration. Mech. Anal. 87, 355-377.

Kloeden, P., Platen, E., 1992. Numerical Solution of Stochastic Differential Equations. Springer, Berlin Heidelberg.

Kruis, F., Kuster, K., 1997. The collision rate of particles in turbulent flow. Chem. Eng. Commun. 158, 201-230.

Laviéville, J., Deutsch, E., Simonin, O., 1995. Large eddy simulation of interaction between colliding particles and a homogeneous isotropic turbulence field. 6th Int. Symp. On Gas-Solid Flows, vol. 228. ASME FED, pp. 347-357.

Laviéville, J., Simonin, O., Berlemont, A., Chang, Z., 1997. Validation of inter-particle collision models based on large eddy simulation in gas-solid turbulent homogeneous shear flow. In: Proc. 7th Int. Symp. on Gas-Particle Flows ASME FEDSM97-3623.

Lun, C., Savage, S., 1986. The effects of an impact velocity dependent coefficient of restitution on stresses developed by sheared granular materials. Acta Mech. 63, 539-559.

Magnaudet, J., Rivero, M., Fabre, J., 1995. Accelerated flows past a rigid sphere or a spherical bubble. Part 1. Steady straining flow. J. Fluid Mech. 284 97-135.

Maxey, M., Riley, J., 1983. Equation of motion for a small rigid sphere in a non uniform flow. Phys. Fluids 26 (4), 2883-2889.

Minier, J., Peirano, E., 2001. The pdf approach to turbulent polydispersed two-phase flows. Phys. Rep. 353, 1-214.

Minier, J.-P., Peirano, E., Chibbaro, S., 2004. Pdf model based on Langevin equation for polydispersed two-phase flows applied to a bluff-body gas-solid flow. Phys. Fluids 16, 2419-2431.

Nanbu, K., 1983. Stochastic solution method of the master equation and the Boltzmann equation. J. Phys. Soc. Jpn. 52, 2654-2658.

O'Rourke, P.J., 1981. Collective Drop Effects in Vaporizing Sprays. Ph.D. Thesis, Princeton University, 1532-T

Pascal, P., Oesterlé, B., 2000. On the dispersion of discrete particles moving in a turbulent shear flow. Int. J. Multiphase Flow 26, 293-325. 
Pialat, X., Simonin, O., Villedieu, P., 2007. A hybrid Eulerian-Lagrangian method to simulate the dispersed phase in turbulent gas-particle flows. Int. J. Multiphase Flow 33 (7), 766-788.

Pigeonneau, F., 1998. Modélisation numérique des collisions de gouttes en écoulements laminaires et turbulents. Ph.D. Thesis, Université Paris VI.

Pope, S., 1994. Lagrangian PDF methods for turbulent flows. Annu. Rev. Fluid Mech. $26,23-63$.

Pope, S., 2002. A stochastic Lagrangian model for acceleration in turbulent flows. Phys. Fluids 14 (7), 2360-2375.

Pope, S., 2002. Stochastic Lagrangian models of velocity in homogeneous turbulent shear flow. Phys. Fluids 14 (5), 1696-1702.

Reade, W., Collins, L., 2000. Effect of preferential concentration on turbulent collisions rates. Phys. Fluids 12, 2530-2540.

Reeks, M.W., 1992. On the continuum equations for dispersed particles in nonuniform flows. Phys. Fluids 4 (6), 1290-1303.

Reeks, M.W., 1993. On the constitutive relations for dispersed particles in nonuniform flows. I: Dispersion in a simple shear flow. Phys. Fluids 5, $750-761$.

Riber, E., Moureau, V., Garcia, M., Poinsot, T., Simonin, O., 2009. Evaluation of numerical strategies for large eddy simulation of particulate two-phase recirculating flows. J. Comput. Phys. 228 (2), 539-564.

Sakiz, M., Simonin, O., 1998. Continuum modelling and Lagrangian simulation of the turbulent transport of particle kinetic stresses in a vertical gas-solid channel flow. In: 3rd International Conference on Multiphase Flows. Lyon, France.

Salazar, J., Jong, J.D., Cao, L., Wooward, S., Meng, H., Collins, L., 2008. Experimental and numerical investigation of inertial particle clustering in isotropic turbulence. J. Fluid Mech. 600, 245-256.

Sawford, B., 1991. Reynolds number effects in Lagrangian stochastic models of turbulent dispersion. Phys. Fluids 3 (6), 1577-1586.

Schiller, L., Nauman, A., 1935. A drag coefficient correlation. V.D.I. Zeitung 77, 318 320.

Shaw, R., Reade, W., Collins, L., Verlinde, J., 1998. Preferential concentration of cloud droplets by turbulence: effects on the early evolution of cumulus cloud droplet spectra. J. Atmos. Sci. 55, 1965-1976.

Sigurgeirsson, H., Stuart, A., Wanz, W.-L., 2001. Algorithms for particle-field simulations with collisions. J. Computat. Phys. 172, 766-807.

Simonin, O., 1996. Combustion and turbulence in two-phase flows. In: Lecture Series 1996-02. Von Karman Institute for Fluid Dynamics.

Simonin, O., Deutsch, E., Minier, J., 1993. Eulerian prediction of the Fluid/Particle correlated motion in turbulent two-phase flows. Appl. Sci. Res. 51, 275-283.

Simonin, O., Février, P., Laviéville, J., 2002. On the spatial distribution of heavy particle velocities in turbulent flow: from continuous field to particulate chaos. J. Turbul. 3 (040), 1-18.

Singh, K., Squires, K.D., Simonin, O., 2004. Evaluation using an LES database of constitutive relations for fluid-particle velocity correlations in fully-developed gas-particle channel flows. In: 5th International Conference on Multiphase Flow. ICMF, Paper No 454, Yokohama, Japan.
Sommerfeld, M., 1999. Inter-particle collisions in turbulent flows: a stochastic lagrangian model. In: Proc. Turbulence and Shear Flow Phenomena -1. Santa Barbara, pp. 265-270.

Sommerfeld, M., 2001. Validation of a stochastic lagrangian modelling approach for inter-particle collisions in homogeneous isotropic turbulence. Int. J. Multiphase Flow 27, 1829-1858.

Squires, K.D., Eaton, J.K., 1991. Preferential concentration of particles by turbulence. Phys. Fluids A: Fluid Dyn. 3 (5), 1169-1178.

Sundaram, S., Collins, L., 1997. Collision statistics in an isotropic particle-laden turbulent suspension. Part 1. Direct numerical simulations. J. Fluid. Mech. 335, 75-109.

Tanaka, T., Tsuji, Y., 1991. Numerical simulation of gas-solid two-phase flow in a vertical pipe on the effects of interparticle collision. 4th Symp. on Gas-Solid Flows, vol. 121. ASME-FED.

Tanière, A., Arcen, B., Oesterlé, B., Pozorski, J., 2010. Study on Langevin model parameters of velocity in turbulent shear flows. Phys. Fluids 22 (11), 115101.

Villedieu, P., Hylkema, J., 1997. Une méthode particulaire aléatoire reposant sur une équation cinétique pour la simulation numérique des sprays denses de gouttelettes liquides. C.R. Acad. Sci. Paris Série I, 323-328.

Villedieu, P., Simonin, O., 2004. Modeling of coalescence in turbulent gas-droplet flows. Commun. Math. Sci. 1, 13-33.

Walton, O., 1993. Numerical simulation of inclined chute flows of monodisperse, inelastic, frictional spheres. Mech. Mater. 16, 239-247.

Williams, J., Crane, R., 1983. Particle collision rate in turbulent flow. Int. J. Multiphase Flow 9 (4), 421-435.

Wunsch, D., Fede, P., Simonin, O., 2008. Development and validation of a binary collision detection algorithm for a polydispersed particle mixture. In: Proc. 8th International Symposium on Numerical Methods for Multiphase Flows, FEDSM2008-55159, pp. 136-144.

Wunsch, D., Fede, P., Simonin, O., 2010a. Validation procedure for discrete particle simulation of turbulent two-phase flows with droplet coalescence. In: ERCOFTAC 82, vol. 82, pp. 40-48.

Wunsch, D. Fede, P. Simonin, O., Villedieu, P. 2010b. Numerical simulation and statistical modeling of inertial droplet coalescence in homogeneous isotropic turbulence. Turbulence and Interactions. Notes on Numerical Fluid Mechanics and Multidisciplinary Design, vol. 110. Springer, Berlin/Heidelberg, pp. 401-407.

Yudine, M., 1959. Physical consideration on heavy-particle dispersion. Adv. Geophys. 6, 185-191.

Zaichik, L., Simonin, O., Alipchenkov, V., 2003. Two statistical models for predicting collision rates of inertial particles in homogeneous isotropic turbulence. Phys. Fluids 15, 2995-3005.

Zaichik, L.I., Simonin, O., Alipchenkov, V.M., 2006. Collision rates of bidisperse inertial particles in isotropic turbulence. Phys. Fluids 18 (3).

Zaichik, L., Fede, P., Simonin, O., Alipchenkov, V., 2009. Statistical models for predicting the effect of bidisperse particle collisions on particle velocities and stresses in homogeneous anisotropic turbulent flows. Int. J. Multiphase Flow 35, 868-878. 